\title{
Finite volume for three-flavour Partially Quenched Chiral Perturbation Theory through NNLO in the meson sector
}

\author{
Johan Bijnens and Thomas Rössler \\ Department of Astronomy and Theoretical Physics, Lund University, \\ Sölvegatan 14A, SE 223-62 Lund, Sweden \\ E-mail: bijnens@thep.lu.se, thomas.roessler@thep.lu.se
}

\begin{abstract}
We present a calculation of the finite volume corrections to meson masses and decay constants in three flavour Partially Quenched Chiral Perturbation Theory (PQChPT) through two-loop order in the chiral expansion for the flavour-charged (or off-diagonal) pseudoscalar mesons. The analytical results are obtained for three sea quark flavours with one, two or three different masses. We reproduce the known infinite volume results and the finite volume results in the unquenched case. The calculation has been performed using the supersymmetric formulation of $\mathrm{PQChPT}$ as well as with a quark flow technique.

Partial analytical results can be found in the appendices. Some examples of cases relevant to lattice QCD are studied numerically. Numerical programs for all results are available as part of the CHIRON package.
\end{abstract}

KEYwords: Lattice QCD, Quark Masses and SM Parameters, Effective field theories, Chiral Lagrangians

ARXIV EPRINT: 1508.07238 


\section{Contents}

1 Introduction 1

2 Partially Quenched Chiral Perturbation Theory 3

2.1 The Lagrangian 3

2.2 The propagator and notation for masses and residues 5

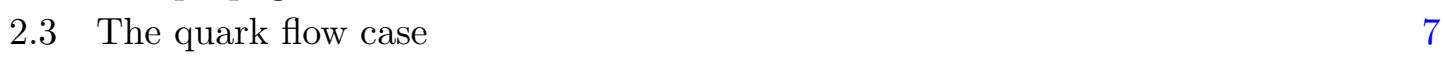

3 The finite volume integrals $\quad 8$

4 Analytical results 10

5 Numerical examples $\quad \mathbf{1 2}$

$5.1 \quad d_{\text {val }}=d_{\text {sea }}=1=1 \quad 12$

$\begin{array}{lll}5.2 & \text { The pion mass } & 12\end{array}$

$\begin{array}{ll}5.3 \text { The pion decay constant } & 14\end{array}$

$\begin{array}{ll}5.4 \text { The kaon mass and decay constant } & 15\end{array}$

$\begin{array}{lll}6 & \text { Conclusions } & 17\end{array}$

$\begin{array}{ll}\text { A Expressions for the mass } & 18\end{array}$

B Expressions for the decay constant $\quad 23$

\section{Introduction}

Quantum chromodynamics (QCD) is nowadays accepted to be the theory describing the strong force. The smallness of the coupling constant at high energies makes it possible to test and confirm the theory in highly energetic scattering. It also provides - at least in principle - a way to obtain various low-energy hadronic observables, such as masses and decay constants, but it has hitherto been impossible to derive such quantities of interest in terms of analytical expressions by means of ab initio calculations. A numerical approach that can circumvent the problem is lattice QCD. A review of the applications to flavour and low-energy hadron physics is [1]. To calculate observables, one uses a numerical evaluation of the QCD path integral in a Monte Carlo approach. A number of restrictions follow from the nature of the calculation. Since it is carried out on a space-time lattice in a finite volume, it is of high interest to have the effect of the finite volume under good control. Furthermore, although lattice computations in meson physics are now feasible when using physical parameters for the light quark masses, a lot of calculations still use unphysically high masses for the quarks. It is also useful to vary quark masses to study a number of 
phenomena. A common solution to study quark mass dependence with lower computational needs is given by partial quenching. In partially quenched QCD (PQQCD), one associates different masses (usually larger ones) to the sea quarks and the valence quarks. Valence quarks are those connected to the external operators while sea quarks are those in the fermion determinant or equivalently in closed loops. Sea quarks are only connected to external states via gluons.

The preferable way to correct for unphysical quark masses is by means of Chiral Perturbation Theory (ChPT) [2-4]. Finite volume effects for ChPT have been introduced in [5-7]. The corresponding effective theory for PQQCD is given by Partially Quenched Chiral Perturbation Theory (PQChPT) [8]. The arguments underlying this are elaborated in [9].

The proper matching of calculations in PQChPT to results from Partially Quenched Lattice QCD allows a whole new landscape of possibilities, such as improved validation and extrapolation of lattice results, or a more accurate determination of the chiral low-energy constants (LECs), see e.g. [10]. It should be stressed that, as opposed to fully quenched calculations, partially quenched calculations are connected to their corresponding unquenched scenarios by a continuous change in variables, making it possible to immediately extract physical results from otherwise unphysical simulations.

In this paper, we address the finite volume corrections through two-loop order in the PQChPT framework, specifically for the flavour-charged or off-diagonal mesons. The infinite volume (IV) results in PQChPT to this order are known for three [11-13] and two [14] sea quark flavours. The finite volume (FV) corrections in (unquenched) ChPT at two-loop order have been addressed in our earlier study [15]. The needed integrals have been worked out in [16]. Our expressions are valid in the frame with vanishing spatial momentum, $\vec{p}=0$, often called the center-of-mass frame. In the so-called moving frames or with twisted boundary conditions there will be additional terms. We have chosen to present our result in terms of lowest order masses given the ambiguity in expressing the results in terms of the large number of possible different physical masses.

Earlier work on finite volume corrections at NNLO are besides our own work [15], the pion mass in two-flavour ChPT [17] and the vacuum expectation value in three flavour ChPT [18]. Extensions of the latter work to partially quenched are in [19]. We did not find published results for the finite volume corrections at one-loop order in the partially quenched case. They are however implicit in the expressions given for the staggered partially quenched case in $[20,21]$.

We give a short list of references for ChPT and discuss some small points in section 2 . The definitions of the integrals we use and how they relate to the results in [16] is given in section 3. The next section describes our major result which is the full finite volume correction to the pion mass and decay constant to two-loop order in ChPT. Section 4 contains the results for the three-flavour case for pion, kaon and eta for both the mass and decay constant. The large two-loop order formulas are collected for one case in the appendices and all of them can be downloaded from [22]. A numerical discussion of our results is in section 5 . 


\section{Partially Quenched Chiral Perturbation Theory}

This section is very similar to the description of PQChPT given in [13] since our work is the extension to finite volume of that paper.

An introduction to ChPT can be found in [23, 24] and in the two-loop review [25]. The lowest order and $p^{4}$-Lagrangian can be found in [4]. The order $p^{6}$ Lagrangian is given in [26]. We use the standard renormalization scheme in ChPT. An extensive discussion of the renormalization scheme can be found in [27] and [28]. Important for our work is that the LECs do not depend on the volume [7]. An introduction with applications to lattice QCD is [29]. References to more introductory literature can be found in [22].

The expansion in ChPT is in momenta $p$ and quark-masses. We count the latter as two powers of $p$. This counting is referred to as $p$-counting. We prefer to designate orders by the $p$-counting order at which the diagram appears. Thus we refer to lowest order (LO) as order $p^{2}$, next-to-leading order (NLO) as order $p^{4}$ or one-loop order and next-to-nextto-leading order (NNLO) as order $p^{6}$ or two-loop order and include in the terminology oneor two-loop order also the diagrams with fewer loops but the same order in $p$-counting.

\subsection{The Lagrangian}

Three massless quark flavours QCD has a chiral symmetry

$$
G=\mathrm{SU}\left(n_{f}\right)_{L} \times \mathrm{SU}\left(n_{f}\right)_{R}
$$

which is spontaneously broken to the diagonal subgroup $\mathrm{SU}(3)_{V}$. The Goldstone bosons following from this spontaneous breakdown are described by the meson octet matrix

$$
\phi(x)=\left(\begin{array}{ccc}
\frac{1}{\sqrt{2}} \pi^{0}+\frac{1}{\sqrt{6}} \eta & \pi^{+} & K^{+} \\
\pi^{-} & -\frac{1}{\sqrt{2}} \pi^{0}+\frac{1}{\sqrt{6}} \eta & K^{0} \\
K^{-} & \bar{K}^{0} & -\frac{1}{\sqrt{3}} \eta
\end{array}\right) .
$$

The flavour-singlet component has been integrated out since it is heavy due to the $\mathrm{U}(1)_{A}$ anomaly. The spontaneous symmetry breaking is the basis of ChPT.

In partially quenched QCD one distinguishes between valence and sea quarks. Valence quarks are connected to the external states (or operators) while the sea-quarks are those contributing in closed loops only connected via gluons to external states. These can be given different masses in lattice QCD calculations. The ChPT for this partial quenching can be done by studying the quark flow generalizing the quenched case studied in [30]. One can then treat the sea and valence lines differently. Alternatively, one can make use of the supersymmetric formulation of PQChPT [8]. In the latter, three corresponding sets of quarks are introduced instead of only two: in addition to the valence and sea sector, a set of so-called ghost quarks is added. These are "bosonic" in the sense that they are treated as commuting variables. With their masses fixed to the same numerical values as present in the valence sector, they will cancel exactly the contribution coming from closed valence quark loops. Most of the remainder of this section will be concerned with the supersymmetric formulation. The changes needed to use a quark flow technique are discussed at the end. 
The chiral symmetry group is formally extended to the graded $^{1}$

$$
G=\mathrm{SU}\left(n_{\mathrm{val}}+n_{\mathrm{sea}} \mid n_{\mathrm{val}}\right)_{L} \times \mathrm{SU}\left(n_{\mathrm{val}}+n_{\mathrm{sea}} \mid n_{\mathrm{val}}\right)_{R}
$$

for the case of $n_{\text {val }}$ valence and $n_{\text {sea }}$ quarks. The chiral group $G$ is spontaneously broken to the diagonal subgroup $\mathrm{SU}\left(n_{\mathrm{val}}+n_{\text {sea }} \mid n_{\mathrm{val}}\right)_{V}$. We will work in the flavour basis rather than in the meson basis. We will thus use fields $\phi_{a b}$ corresponding to the flavour content of $q_{a} \bar{q}_{b}$. The mixing of the neutral eigenstates and the integrating out of the singlet degree of freedom is taken care of by using a more complicated propagator. ${ }^{2}$

The corresponding Goldstone degrees of freedom are put in a matrix with the generic structure

$$
\Phi=\left(\begin{array}{l}
{\left[q_{V} \bar{q}_{V}\right]\left[q_{V} \bar{q}_{S}\right]\left[q_{V} \bar{q}_{B}\right]} \\
{\left[q_{S} \bar{q}_{V}\right]\left[q_{S} \bar{q}_{S}\right]\left[q_{S} \bar{q}_{B}\right]} \\
{\left[q_{B} \bar{q}_{V}\right]\left[q_{B} \bar{q}_{S}\right]\left[q_{B} \bar{q}_{B}\right]}
\end{array}\right) .
$$

$V$ denotes valence, $S$ denotes sea and $B$ denotes the bosonic ghost quarks. Note that the meson fields containing one single ghost quark only will themselves obey fermionic, i.e. anticommuting, statistics.

The structure of the Lagrangian is similar to standard ChPT for a generic number of flavours. The lowest order Lagrangian is

$$
\mathcal{L}_{2}=\frac{F_{0}^{2}}{4}\left\langle u_{\mu} u^{\mu}+\chi_{+}\right\rangle .
$$

At one-loop, it is given by

$$
\begin{aligned}
\mathcal{L}_{4}= & \hat{L}_{0}\left\langle u^{\mu} u^{\nu} u_{\mu} u_{\nu}\right\rangle+\hat{L}_{1}\left\langle u^{\mu} u_{\mu}\right\rangle^{2}+\hat{L}_{2}\left\langle u^{\mu} u^{\nu}\right\rangle\left\langle u_{\mu} u_{\nu}\right\rangle+\hat{L}_{3}\left\langle\left(u^{\mu} u_{\mu}\right)^{2}\right\rangle \\
& +\hat{L}_{4}\left\langle u^{\mu} u_{\mu}\right\rangle\left\langle\chi_{+}\right\rangle+\hat{L}_{5}\left\langle u^{\mu} u_{\mu} \chi_{+}\right\rangle+\hat{L}_{6}\left\langle\chi_{+}\right\rangle^{2}+\hat{L}_{7}\left\langle\chi_{-}\right\rangle^{2}+\frac{\hat{L}_{8}}{2}\left\langle\chi_{+}^{2}+\chi_{-}^{2}\right\rangle+\ldots
\end{aligned}
$$

We show only the terms relevant for our work.

The generalized Goldstone manifold is parametrized by

$$
u \equiv \exp (i \Phi /(\sqrt{2} \hat{F}))
$$

similar to the exponential representation in standard ChPT. It is a $9 \times 9$ matrix with fermionic parts. We have furthermore introduced

$$
\begin{aligned}
u_{\mu} & =i\left\{u^{\dagger}\left(\partial_{\mu}-i r_{\mu}\right) u-u\left(\partial_{\mu}-i l_{\mu}\right) u^{\dagger}\right\} \\
\chi_{ \pm} & =u^{\dagger} \chi u^{\dagger} \pm u \chi^{\dagger} u .
\end{aligned}
$$

\footnotetext{
${ }^{1}$ The precise structure of the symmetry group is somewhat different, but the one given here is sufficient for both the present discussion as well as for practical calculations. The "approximate" symmetry group reproduces the right Ward identities [10, 31].

${ }^{2}$ This is described in detail in [31]. It is possible to use the same method also in standard ChPT.
} 
The matrix $\chi$ is for this work restricted to

$$
\chi=2 B_{0} \operatorname{diag}\left(m_{1}, \ldots, m_{9}\right)
$$

with $m_{i}$ the quark mass of quark $i$ and $B_{0}$ a LEC. We have here $m_{1}=m_{7}, m_{2}=m_{8}, m_{3}=$ $m_{9}$ as the valence masses and $m_{4}, m_{5}, m_{6}$ as the sea quark masses. Ordinary traces have been replaced by supertraces, denoted by \langle\rangle , defined in terms of the ordinary ones by

$$
\operatorname{Str}\left(\begin{array}{ll}
A & B \\
C & D
\end{array}\right)=\operatorname{Tr} A-\operatorname{Tr} D
$$

$B$ and $C$ denote the fermionic blocks in the matrix. The supersinglet $\Phi_{0}$, generalizing the $\eta^{\prime}$, is integrated out to account for the axial anomaly as in standard ChPT, implying the additional condition

$$
\langle\Phi\rangle=\operatorname{Str}(\Phi)=0
$$

However, as mentioned above, we will work in the flavour basis enforcing the constraint (2.11) via the propagator.

A calculation in PQChPT has to be performed using a larger set of operators since no further reduction by means of Cayley-Hamilton relations can be performed. The threeflavour PQChPT Lagrangian (equation (2.6)) thus has 11 LECs for PQChPT.

The LECs for standard three flavour ChPT are related to those of three flavour PQChPT via

$$
L_{1}^{r}=\hat{L}_{1}^{r}+\hat{L}_{0}^{r} / 2, \quad L_{2}^{r}=\hat{L}_{2}^{r}+\hat{L}_{0}^{r}, \quad L_{3}^{r}=\hat{L}_{3}^{r}-2 \hat{L}_{0}^{r}
$$

and $L_{i}^{r}=\hat{L}_{i}^{r}$ for the others. Note that a numerical value for $\hat{L}_{0}$ cannot be obtained by experiment, but can be determined only via PQQCD lattice simulations or modelling.

An additional comment is that the divergences for $\mathrm{PQChPT}$ are directly related to

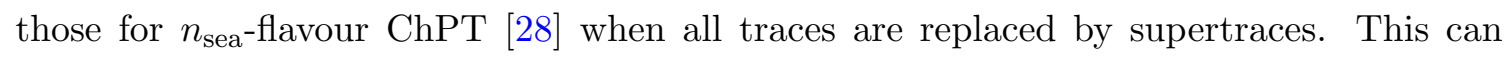
be argued using the formal equivalence of the equations of motion used or via the replica trick [32].

\subsection{The propagator and notation for masses and residues}

The variant of $\mathrm{PQChPT}$, considered in this paper, comes with three valence quarks, with masses $m_{1}, m_{2}, m_{3}$ and three sea quarks with masses $m_{4}, m_{5}, m_{6}$. The additional ghost quarks emerging only in the supersymmetric formulation have masses $m_{7}, m_{8}, m_{9}$. They do not appear explicitly since they are fixed to the ones in the valence sector, i.e. $m_{7}=m_{1}$, $m_{8}=m_{2}, m_{9}=m_{3}$.

We use the numbers $d_{\text {val }}$ and $d_{\text {sea }}$ to denote the number of non-degenerate quark masses in each sector. In the case of two non-degenerate mass scales for one sector, it is the two masses with the lowest indices that we set degenerate, which will in turn both be represented by the mass scale with the lowest index, e.g. in the case $d_{\text {sea }}=2$ we have $m_{4}=m_{5} \neq m_{6}$ and expressions will be explicitly dependent on $m_{4}$ and $m_{6}$ only. 
In fact we will always absorb a factor $2 B_{0}$ in the notation and we use

$$
\chi_{i} \equiv 2 B_{0} m_{i}, \quad \chi_{i j} \equiv \frac{1}{2}\left(\chi_{i}+\chi_{j}\right) .
$$

The lowest order masses for off-diagonal mesons with flavour content $q_{i} \bar{q}_{j}$ are given by $\chi_{i j}$ and we will use $\chi_{i}$ rather then $\chi_{i i}$ for equal masses. Dealing with masses for the diagonal valence mesons in PQChPT is not trivial. This is discussed in detail in [31] and extended to NNLO in [33]. The diagonal sea quark sector has two masses associated with it, corresponding to the neutral pion and eta masses. These we denote by $\chi_{\pi}$ and $\chi_{\eta}$. They are defined as the solutions to the equations

$$
\begin{aligned}
\chi_{\pi}+\chi_{\eta} & =\frac{2}{3}\left(\chi_{4}+\chi_{5}+\chi_{6}\right), \\
\chi_{\pi} \chi_{\eta} & =\frac{1}{3}\left(\chi_{4} \chi_{5}+\chi_{5} \chi_{6}+\chi_{4} \chi_{6}\right) .
\end{aligned}
$$

They are non-polynomial in the sea masses $\chi_{j}$ for three non-degenerate quark masses, i.e. $d_{\text {sea }}=3$. For $d_{\text {sea }}=2$ one has instead $\chi_{\pi}=\chi_{4}$ and $\chi_{\eta}=(1 / 3)\left(\chi_{4}+2 \chi_{6}\right)$.

The flavour-charged propagator, connecting $\phi_{i j}$ with $\phi_{j i}$, is given by $[8,10,31]$

$$
-i G_{i j}^{c}(k)=\frac{\epsilon_{j}}{k^{2}-\chi_{i j}+i \varepsilon} \quad(i \neq j),
$$

with $\chi_{i j} \equiv\left(\chi_{i}+\chi_{j}\right) / 2$, the lowest order meson mass, and the signature $\epsilon_{j}$ is defined as +1 for the flavor indices of the $n_{\text {val }}+n_{\text {sea }}$ fermionic quarks, and as -1 for the flavor indices of the $n_{\text {val }}$ bosonic ghost quarks. In the present calculation, with the number of valence and sea quarks as given above, $\epsilon_{j}$ thus takes the values

$$
\epsilon_{j}=\left\{\begin{array}{l}
+1 \text { for } j=1, \ldots, 6 \\
-1 \text { for } j=7,8,9 .
\end{array}\right.
$$

The flavour-neutral propagator, connecting a flavour field $\phi_{i i}$ to $\phi_{j j}$, on the other hand suffers from additional contributions emerging from the elimination of the $\Phi_{0}$ and the partial quenching $[8,10,31]$. We write it as

$$
G_{i j}^{n}(k)=G_{i j}^{c}(k) \delta_{i j}-G_{i j}^{q}(k) / n_{\text {sea }} .
$$

The additional terms are either

$$
\begin{aligned}
-i G_{i j}^{q}(k)= & \frac{R_{j \pi \eta}^{i}}{k^{2}-\chi_{i}+i \varepsilon}+\frac{R_{i \pi \eta}^{j}}{k^{2}-\chi_{j}+i \varepsilon} \\
& +\frac{R_{\eta i j}^{\pi}}{k^{2}-\chi_{\pi}+i \varepsilon}+\frac{R_{\pi i j}^{\eta}}{k^{2}-\chi_{\eta}+i \varepsilon}
\end{aligned}
$$

for the case with $i \neq j$ and $\chi_{i} \neq \chi_{j}$, or

$$
\begin{aligned}
-i G_{i j}^{q}(k)= & \frac{R_{i}^{d}}{\left(k^{2}-\chi_{i}+i \varepsilon\right)^{2}}+\frac{R_{i}^{c}}{k^{2}-\chi_{i}+i \varepsilon} \\
& +\frac{R_{\eta i i}^{\pi}}{k^{2}-\chi_{\pi}+i \varepsilon}+\frac{R_{\pi i i}^{\eta}}{k^{2}-\chi_{\eta}+i \varepsilon},
\end{aligned}
$$


for the case with $\chi_{i}=\chi_{j}$ which clearly includes $i=j$. In the second case, the sum of single poles is supplemented with an unphysical double pole. Since double poles emerge due to the partial quenching in the valence sector, they disappear by taking the appropriate unquenched limit.

Using the ratios of products of differences of masses

$$
\begin{aligned}
R_{a b}^{z} & =\chi_{a}-\chi_{b}, \\
R_{a b c}^{z} & =\frac{\chi_{a}-\chi_{b}}{\chi_{a}-\chi_{c}} \\
R_{a b c d}^{z} & =\frac{\left(\chi_{a}-\chi_{b}\right)\left(\chi_{a}-\chi_{c}\right)}{\chi_{a}-\chi_{d}}, \\
R_{a b c d e f g}^{z} & =\frac{\left(\chi_{a}-\chi_{b}\right)\left(\chi_{a}-\chi_{c}\right)\left(\chi_{a}-\chi_{d}\right)}{\left(\chi_{a}-\chi_{e}\right)\left(\chi_{a}-\chi_{f}\right)\left(\chi_{a}-\chi_{g}\right)},
\end{aligned}
$$

the residues $R$ of the neutral meson propagator in equations (2.18) and (2.19) are (for $\left.d_{\text {sea }}=3\right)$

$$
R_{j k l}^{i}=R_{i 456 j k l}^{z}, \quad R_{i}^{d}=R_{i 456 \pi \eta}^{z}, \quad R_{i}^{c}=R_{4 \pi \eta}^{i}+R_{5 \pi \eta}^{i}+R_{6 \pi \eta}^{i}-R_{\pi \eta \eta}^{i}-R_{\pi \pi \eta}^{i} .
$$

Note that many of these quantities vanish when $i$ takes the value of a sea quark index. The sea-quark propagators thus do not contribute any double poles as expected since these originate from the quenching in the valence sector.

For $d_{\text {sea }}=2$ or $\chi_{\pi}=\chi_{5}=\chi_{4}$. The needed residues simplify to

$$
R_{j k}^{i}=R_{i 46 j k}^{z}, \quad R_{i}^{d}=R_{i 46 \eta}^{z}, \quad R_{i}^{c}=R_{4 \eta}^{i}+R_{6 \eta}^{i}-R_{\eta \eta}^{i} .
$$

The corresponding propagator can be obtained by removing all pion indices as well as the pion mass pole from equations (2.18) and (2.19).

The physically less interesting case $d_{\text {sea }}=1$ immediately yields $\chi_{\pi}=\chi_{\eta}=\chi_{6}=\chi_{5}=\chi_{4}$. All residues from the sea quark sector are reduced to numbers, only

$$
R_{j}^{i}=R_{i 4 j}^{z}, R_{i}^{d}=R_{i 4}^{z},
$$

appear.

\subsection{The quark flow case}

We have performed the calculation using the supersymmetric method described above but also with the quark flow method [30]. We use the same Lagrangians as in (2.5) and (2.6) but with normal traces everywhere. The matrix $\Phi$ is now written in terms of generic fields $\phi_{i j}$ and all indices are kept symbolic implying summations.

Connecting propagators of a field $\phi_{i j}$ to $\phi_{k l}$ should be done by using

$$
G_{i j k l}(k)=G_{i j}^{c}(k) \delta_{i l} \delta_{j k}-\delta_{i j} \delta_{k l} G_{i k}^{q}(k) / n_{\text {sea }} .
$$

The propagators $G_{i j}^{c}(k), G_{i j}^{q}(k)$ remain the same but we can now disregard the factors $\epsilon_{j}$ since with this method there are no bosonic ghost quarks. 
After constructing the Feynman diagrams using the above, the quark flow is visible following the symbolic flavour indices. Next, one replaces the index lines that connect to external fields or operators by their appropriate valence value. The remaining index lines are now sea indices and are summed over with the sea quark indices.

The results obtained with the quark flow method agreed in all cases with those of the supersymmetric method.

\section{The finite volume integrals}

The loop integrals at finite volume at one-loop are well known. There is a sum over discrete momenta in every direction with a finite size rather than a continuous integral. The Poisson summation formula allows to identify the infinite volume part and the finite volume corrections. The remainder can be done with two different methods. For one-loop tadpole integrals the first method was introduced by [5-7] and a sum over Bessel functions, that for large $M L$ converges fast, remains to be done. With the other method one remains instead with an integral over a Jacobi theta function, this method can be used for small and medium $M L$ as well. It can be found in [34]. The extensions to other one-loop integrals is done in both cases by combining propagators with Feynman parameters. The first method was extended to the equal mass two-loop sunset integral [17] and later to the more general mass case in [16]. The latter extended the Jacobi theta function method as well to the sunset case. Details and further references can be found in [16]. In this paper we use Minkowski notation for the integrals.

For the one-loop integrals needed here, we use a notation that does a first classification according to the sum of the powers of the propagators with different masses, $m_{1}, m_{2}, \ldots, m_{\max }$. We label the integrals $A, B, C, D$ for a total power of propagators of $n=1,2,3,4$ respectively, since total powers of up to 4 can appear in the calculation as follows from the discussion of double poles in section 2.2. The different mass scales are given as consecutive arguments of the integral. Alternatively, if only one mass scale in total is present, we omit its repetition as a shorthand notation. For the present calculation at most two different scales can appear.

Both scalar and tensor integrals will occur, e. g. in the simplest case of one single propagator raised to single power

$$
\left\{A\left(m^{2}\right), A_{\mu \nu}\left(m^{2}\right)\right\}=\frac{1}{i} \int_{V} \frac{d^{d} r}{(2 \pi)^{d}} \frac{\left\{1, r_{\mu} r_{\nu}\right\}}{\left(r^{2}-m^{2}\right)} .
$$

We used the subscript $V$ to indicate it is a finite volume sum and integral.

More Lorentz structures are possible than in the infinite volume case. We define the tensor $t_{\mu \nu}$ as the spatial part of the Minkowski metric $g_{\mu \nu}$, to express these. For the centerof-mass (cms) case this is sufficient. The needed functions for the above example are

$$
A_{\mu \nu}\left(m^{2}\right)=g_{\mu \nu} A_{22}\left(m^{2}\right)+t_{\mu \nu} A_{23}\left(m^{2}\right) .
$$

We then use Passarino-Veltman identities in order to further simplify the result. In infinite volume the relation obtained by considering $g^{\mu \nu} A_{\mu \nu}\left(m^{2}\right)$ can be used to remove $A_{22}$. In 


\begin{tabular}{|c|ccc|}
\hline & $n_{1}$ & $n_{2}$ & $n_{3}$ \\
\hline$n=1$ & 1 & 1 & 1 \\
\hline$n=2$ & 2 & 1 & 1 \\
$n=3$ & 1 & 2 & 1 \\
$(n=4)$ & 1 & 1 & 2 \\
\hline$n=5$ & 2 & 2 & 1 \\
$(n=6)$ & 2 & 1 & 2 \\
$n=7$ & 1 & 2 & 2 \\
\hline$n=8$ & 2 & 2 & 2 \\
\hline
\end{tabular}

Table 1. Overview of the notation for the possible configurations of powers of propagators in the $H$ functions in PQChPT. Redundant configurations are given in parentheses.

finite volume, we again remove the $A_{22}$-type integrals from the extended relation

$$
d A_{22}\left(m^{2}\right)+3 A_{23}\left(m^{2}\right)=m^{2} A\left(m^{2}\right) .
$$

Each integral is split into an infinite volume contribution and a finite volume correction by means of the Poisson summation formula, while simultaneously being expanded in $\epsilon$ up to the necessary order.

$$
A\left(m^{2}\right)=\lambda_{0} \frac{m^{2}}{16 \pi^{2}}+\bar{A}\left(m^{2}\right)+A^{V}\left(m^{2}\right)+\epsilon\left(A^{\epsilon}\left(m^{2}\right)+A^{V \epsilon}\left(m^{2}\right)\right)+\cdots .
$$

Here, $\lambda_{0}=\frac{1}{\epsilon}+\log (4 \pi)+1-\gamma$. The same split is done for all one-loop integrals. The expressions can be obtained by using the relations

$$
\begin{aligned}
B\left(m^{2}\right) & =\frac{\partial}{\partial m^{2}} A\left(m^{2}\right), \\
C\left(m^{2}\right) & =\frac{1}{2} \frac{\partial}{\partial m^{2}} B\left(m^{2}\right), \\
D\left(m^{2}\right) & =\frac{1}{3} \frac{\partial}{\partial m^{2}} C\left(m^{2}\right), \\
B\left(m_{1}^{2}, m_{2}^{2}\right) & =\frac{A\left(m_{1}^{2}\right)-A\left(m_{2}^{2}\right)}{m_{1}^{2}-m_{2}^{2}} .
\end{aligned}
$$

The sunset integrals, defined as

$$
\begin{aligned}
& \left\{H, H_{\mu}, H_{\mu}^{s}, H_{\mu \nu}, H_{\mu \nu}^{r s}, H_{\mu \nu}^{s s}\right\}\left(n, m_{1}^{2}, m_{2}^{2}, m_{3}^{2}, p\right)= \\
& \quad \frac{1}{i^{2}} \int_{V} \frac{d^{d} r}{(2 \pi)^{d}} \frac{d^{d} s}{(2 \pi)^{d}} \frac{\left\{1, r_{\mu}, s_{\mu}, r_{\mu} r_{\nu}, r_{\mu} s_{\nu}, s_{\mu} s_{\nu}\right\}}{\left(r^{2}-m_{1}^{2}\right)^{n_{1}}\left(s^{2}-m_{2}^{2}\right)^{n_{2}}\left((r+s-p)^{2}-m_{3}^{2}\right)^{n_{3}}},
\end{aligned}
$$

now come with eight different pole configurations. We label these by the index $n$ according to table 1 analoguous to the infinite volume definitions of [11-14].

The interchange $\left(r, m_{1}^{2}, n_{1}\right) \leftrightarrow\left(s, m_{2}^{2}, n_{2}\right)$ allows to show that $H_{\mu}^{s}, H_{\mu \nu}^{s s}$ are related directly to $H_{\mu}^{r}, H_{\mu \nu}^{r r}$. $H_{\mu \nu}^{r s}$ can also be related to $H_{\mu \nu}$ using the trick shown in [35] and 
also used in [16], now taking the pole configurations into account properly. The resulting $H_{\mu \nu}$ and $H_{\mu}$ can then be reduced to six pole configurations only, cf. table 1 , the bracketed ones can be eliminated via the interchange above. In the scalar case $H$, only four pole configurations are needed.

For the partially quenched calculation we thus generalized the sunset integrals used in our earlier work via

$$
H\left(\chi_{i}, \chi_{j}, \chi_{k} ; p^{2}\right) \rightarrow H\left(n, \chi_{i}, \chi_{j}, \chi_{k} ; p^{2}\right),
$$

introducing the new index $n$ for the pole configurations as the first argument. Note on the side that all new pole configurations are related to the simplest one by differentiation with respect to the mass scales.

In the cms frame, we reduce the tensor structure of the sunsets as

$$
\begin{aligned}
H_{\mu} & =p_{\mu} H_{1} \\
H_{\mu \nu} & =p_{\mu} p_{\nu} H_{21}+g_{\mu \nu} H_{22}+t_{\mu \nu} H_{27} .
\end{aligned}
$$

As in [15], we renormalize the FV sunsets by not only subtracting the infinite part but also an additional finite part containing $\mathcal{O}(\epsilon)$ contributions of one-loop integrals. In this way, the latter integrals will cancel out of the final result, and thus do not need to be computed. The splitting for $n=1$

$$
\begin{aligned}
\tilde{H}^{V}= & \frac{\lambda_{0}}{16 \pi^{2}}\left(A^{V}\left(m_{1}^{2}\right)+A^{V}\left(m_{2}^{2}\right)+A^{V}\left(m_{3}^{2}\right)\right)+\frac{1}{16 \pi^{2}}\left(A^{V \epsilon}\left(m_{1}^{2}\right)+A^{V \epsilon}\left(m_{2}^{2}\right)+A^{V \epsilon}\left(m_{3}^{2}\right)\right) \\
& +H^{V} \\
\tilde{H}_{1}^{V}= & \frac{\lambda_{0}}{16 \pi^{2}} \frac{1}{2}\left(A^{V}\left(m_{2}^{2}\right)+A^{V}\left(m_{3}^{2}\right)\right)+\frac{1}{16 \pi^{2}} \frac{1}{2}\left(A^{V \epsilon}\left(m_{2}^{2}\right)+A^{V \epsilon}\left(m_{3}^{2}\right)\right)+H_{1}^{V} \\
\tilde{H}_{21}^{V}= & \frac{\lambda_{0}}{16 \pi^{2}} \frac{1}{3}\left(A^{V}\left(m_{2}^{2}\right)+A^{V}\left(m_{3}^{2}\right)\right)+\frac{1}{16 \pi^{2}} \frac{1}{3}\left(A^{V \epsilon}\left(m_{2}^{2}\right)+A^{V \epsilon}\left(m_{3}^{2}\right)\right)+H_{21}^{V} \\
\tilde{H}_{27}^{V}= & \frac{\lambda_{0}}{16 \pi^{2}}\left(A_{23}^{V}\left(m_{1}^{2}\right)+\frac{1}{3} A_{23}\left(m_{2}^{2}\right)+\frac{1}{3} A_{23}^{V}\left(m_{3}^{2}\right)\right) \\
& +\frac{1}{16 \pi^{2}}\left(A_{23}^{V \epsilon}\left(m_{1}^{2}\right)+\frac{1}{3} A_{23}^{V \epsilon}\left(m_{2}^{2}\right)+\frac{1}{3} A_{23}^{V \epsilon}\left(m_{3}^{2}\right)\right)+H_{27}^{V}
\end{aligned}
$$

has to be generalized for the other pole configurations by taking the appropriate derivatives w.r.t. the masses.

\section{Analytical results}

The calculation of the masses proceeds in the usual way from the Feynman diagrams for the self-energy shown in figure 1. We have performed the calculation for the off-diagonal mesons, i.e. consistening of a valence quark and a different valence anti-quark, and for the case of three flavours of sea quarks. The calculation has been done for all mass cases, equal and different valence quark-masses, $d_{\mathrm{val}}=1,2$, and sea quark masses all equal, $d_{\text {sea }}=1$, two equal and the third different, $d_{\text {sea }}=2$ and all three different, $d_{\text {sea }}=3$.

A large number of checks have been done on the calculations. They have been performed both in the supersymmetric formalism and using quark flow techniques. The infinite 

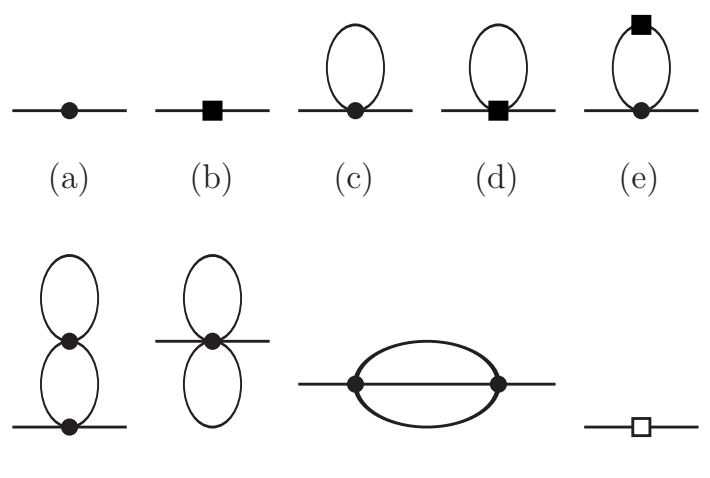

(f) (g) (h) $\quad$ (i)

Figure 1. Diagrammatic contributions to the pseudoscalar self-energy, up to $\mathcal{O}\left(p^{6}\right)$. Circular vertices are of $\mathcal{O}\left(p^{2}\right)$, the filled boxes are of $\mathcal{O}\left(p^{4}\right)$, the open box is of $\mathcal{O}\left(p^{6}\right)$. The tree level diagrams $(\mathrm{a}, \mathrm{b}, \mathrm{i})$ do not contribute to finite volume corrections.

volume results are also in full agreement with [11-13]. The finite volume parts agree with our earlier results [15] when these are expressed in terms of lowest order masses and when the sea masses are put equal to the valence masses.

The formulas especially for the case of three different sea quark masses are very long. In appendix A we list the case of equal valence masses and two sea quark masses. This corresponds to the charged pion mass in the isospin limit. The other cases can be downloaded from [22].

The masses are given as

$$
m_{i j}^{2}=\chi_{i j}+m_{i j}^{2(4)}+\Delta^{V} m_{i j}^{2(4)}+m_{i j}^{2(6)}+\Delta^{V} m_{i j}^{2(6)} .
$$

In addition a superscript indicating $d_{\text {val }} d_{\text {sea }}$ is added. The infinite volume and the oneloop finite volume corrections were known before. The new parts are the two-loop finite volume corrections. These we split in addition in an $L_{i}^{r}$ dependent part and a pure two-loop contribution

$$
\Delta^{V} m_{i j}^{2(6)}=\Delta^{V} m_{i j}^{2(6 L)}+\Delta^{V} m_{i j}^{2(6 R)} .
$$

The subscript $i j$ is set to 12 for $d_{\mathrm{val}}=1$ and to 13 for $d_{\mathrm{val}}=2$ similar to the infinite volume work.

The decay constant is defined in the usual way as

$$
\left\langle 0\left|\bar{q}_{j} \gamma_{\mu} \gamma_{5} q_{i}\right| M_{i j}(p)\right\rangle=i \sqrt{2} F_{i j} p_{\mu},
$$

for the pseudoscalar meson $M_{i j}$ with quark content $i \neq j$ and momentum $p$. The calculation needs the the diagrams of figure 1 for the wave function renormalization and the same ones with one external meson leg replaced by an insertion of the axial current.

We split the result as

$$
F_{i j}=F_{0}+F_{i j}^{(4)}+\Delta^{V} F_{i j}^{(4)}+F_{i j}^{(6)}+\Delta^{V} F_{i j}^{(6)} .
$$

The NNLO part is split again in

$$
\Delta^{V} F_{i j}^{(6)}=\Delta^{V} F_{i j}^{(6 L)}+\Delta^{V} F_{i j}^{2(6 R)} .
$$


The calculations have been done using the supersymmetric and the quark flow methods. The infinite volume and NLO results agree with the known expressions and the result reduces in the correct limit to the unquenched results of our earlier work [15]. The formulas are rather long, the case for equal valence masses and two different sea masses corresponding to the charged pion decay constant in the isospin limit is given in appendix B. The expressions for the other cases can be downloaded from [22].

\section{$5 \quad$ Numerical examples}

The intention is that various lattice QCD collaborations can use our formulas. All cases discussed have been included in the package CHIRON [36] available from [37]. The numerical results shown in this section have been obtained with that implementation. The programs have been cross-checked with an independent version. It has been checked that the results reduce in the appropriate limits to those of our earlier work [15]. For this purpose the expressions obtained in [15], but rewritten in terms of lowest order masses and decay constants, have been implemented and included in CHIRON [37]. In addition, a check has been done that the different mass cases reduce to each other numerically.

For input values we have chosen the recent global fit for the $L_{i}^{r}$ [38]. We have set the extra LEC $L_{0}^{r}=0$. We always use a scale of $\mu=0.77 \mathrm{GeV}$. For the size of the lattice we present results for a length $L$ such that $M L=2$ for $M=0.13 \mathrm{GeV}$. The lowest order pion decay constant we have chosen throughout as $F_{0}=87.7 \mathrm{MeV}$.

The numerical results are presented via

$$
\begin{aligned}
\Delta_{M}^{V} & =\frac{m_{i j}^{2 V}-m_{i j}^{2 \infty}}{\chi_{i j}} \\
\Delta_{F}^{V} & =\frac{F_{i j}^{V}-F_{i j}^{\infty}}{F_{0}} .
\end{aligned}
$$

We thus plot the size of the finite volume corrections relative to the lowest order value of the quantity under consideration. Note that the results are for charged or off-diagonal mesons. They consist of a quark and a different anti-quark which might have equal mass.

\section{$5.1 \quad d_{\text {val }}=d_{\text {sea }}=1=1$}

Here we set all valence and all sea masses equal, $d_{\text {val }}=d_{\text {sea }}=1$. The size of the finite volume corrections as a function of $\chi_{1}$ and $\chi_{4}$ is shown in figure 2. The corrections in this case are reasonable, at most a few $\%$, except for very low masses and become very large for low valence and high sea quark mass.

\subsection{The pion mass}

In this subsection we look at the case where the lowest order mass is around the pion mass. We plot $\Delta_{M}^{V}$ with $\sqrt{\chi_{12}}=0.13 \mathrm{GeV}$. The strange sea quark mass we have always chosen such that the average lowest order kaon mass is $0.45 \mathrm{GeV}$. This corresponds to $\sqrt{\chi_{6}}=\sqrt{2(0.45)^{2}-(0.13)^{2}} \mathrm{GeV} \approx 0.623 \mathrm{GeV}$. The other input parameters are chosen as 

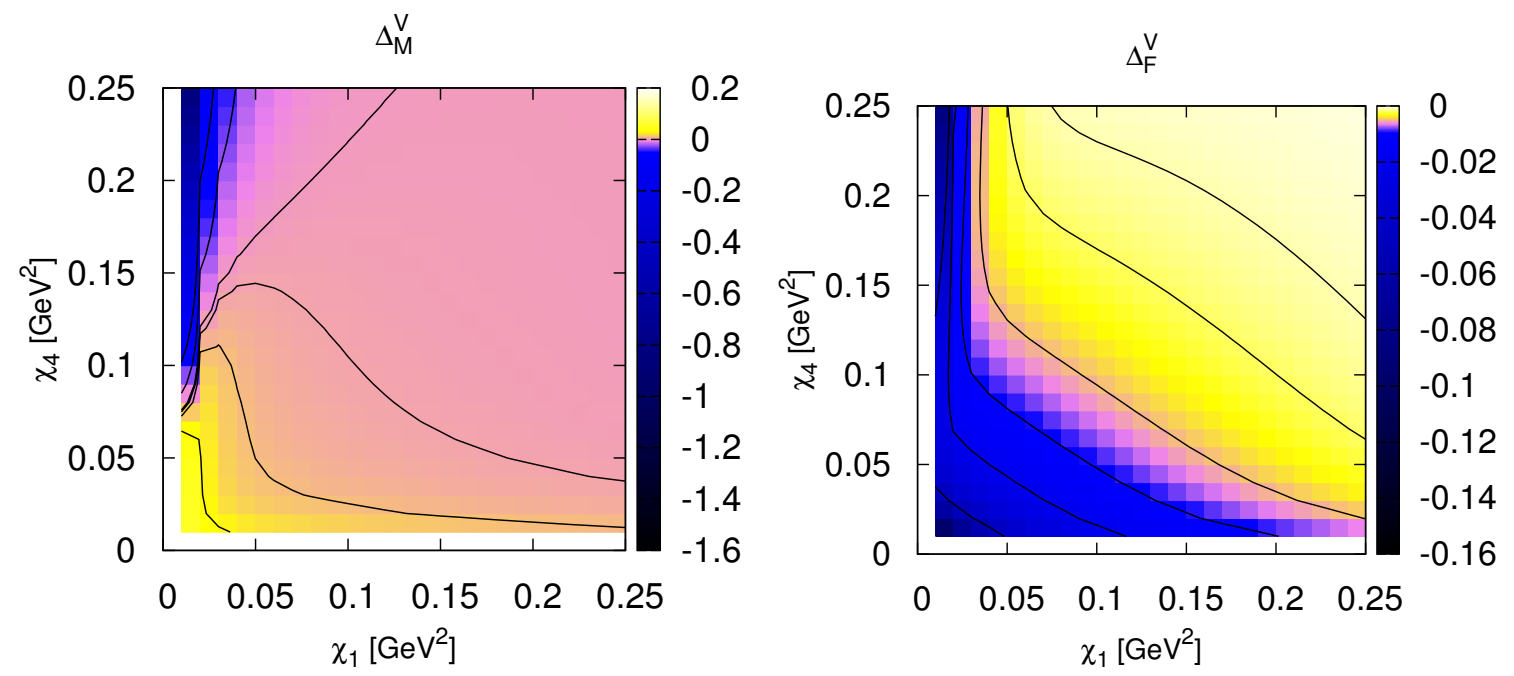

Figure 2. The finite volume corrections relative to the lowest order value as defined in (5.1) for the case with all valence masses equal and all sea masses equal. Left: $\Delta_{M}^{V}$ the correction to the mass-squared, contour lines are drawn at $0.03,0.01,0.003,0,-0.03,-0.01$ starting from the bottom left and going counterclockwise. Right: the correction to the decay constant, contour lines are drawn at $-0.001,-0.002,-0.005,-0.01,-0.02,-0.05$ going from top-right to bottom-left.

given in the introduction of this section. We have restricted the sea up and down quark masses corresponding to a lowest order sea quark pion mass of 100 to $300 \mathrm{MeV}$.

The first case we look at is $d_{\text {val }}=1, d_{\text {sea }}=2$. This corresponds to taking the up and down quark masses equal in both the valence and sea quark sector and a different strange quark mass. This is the isospin limit. The result is shown in figure 3(a). There is a rather large cancellation between the $p^{4}$ and $p^{6}$ correction while the $p^{6}$ contribution coming from the $L_{i}^{r}$ is fairly small.

We now include isospin breaking in the valence sector. We thus look at the case with $d_{\text {val }}=2, d_{\text {sea }}=2$. We fix the valence quark masses such that $\chi_{1}+\chi_{2}=2 \chi_{12}$ and $\chi_{1} / \chi_{2}=1 / 2$. There is a sizable isospin breaking visible in the finite volume corrections, as shown in figure $3(\mathrm{~b})$.

The opposite case, isospin breaking in the sea sector, but not in the valence sector, leads to numerically similar but opposite sign corrections. Here we used $\chi_{1}=\chi_{2}, \chi_{4}=\chi_{5} / 2$ and $\chi_{4}+\chi_{5}=2 \chi_{\mathrm{av}}$. The results are shown in figure 3(c).

Finally, we introduce isospin breaking in both the valence and sea quark sector with $\chi_{1} / \chi_{2}=1 / 2, \chi_{4}=\chi_{5} / 2$ and $\chi_{4}+\chi_{5}=2 \chi_{\mathrm{av}}$. The results are shown in figure $3(\mathrm{~d})$. The total isospin corrections are rather small.

The numerical cancellation between the isospin breaking in the valence and sea quark case is accidental. The corrections due to valence and sea quark masses are all second order in isopin breaking. The same argument as in the unquenched case goes through both for the valence and sea quark masses. We have compared four scenarios in figure 4 . We show the $p^{4}$ and the full $p^{4}+p^{6}$ result first with no isospin breaking, then only in the valence sector or only in the sea sector and finally in both sectors. The curves are those shown 


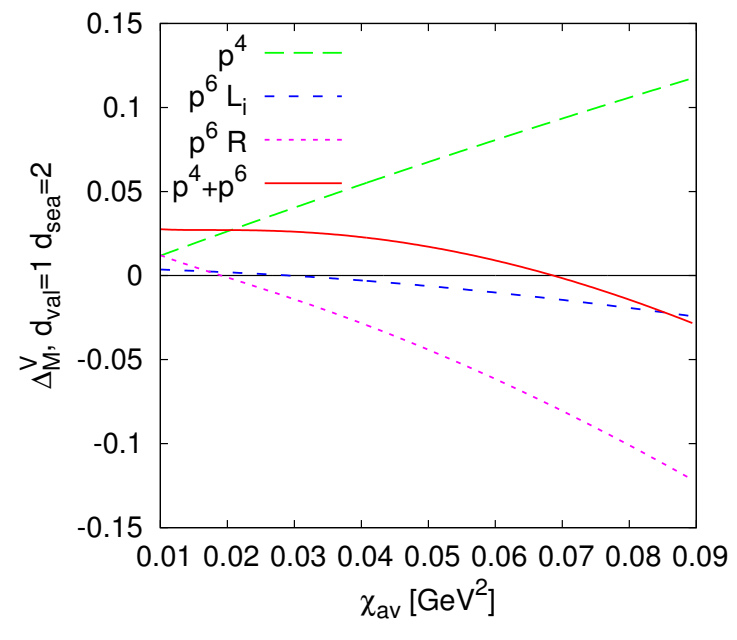

(a)

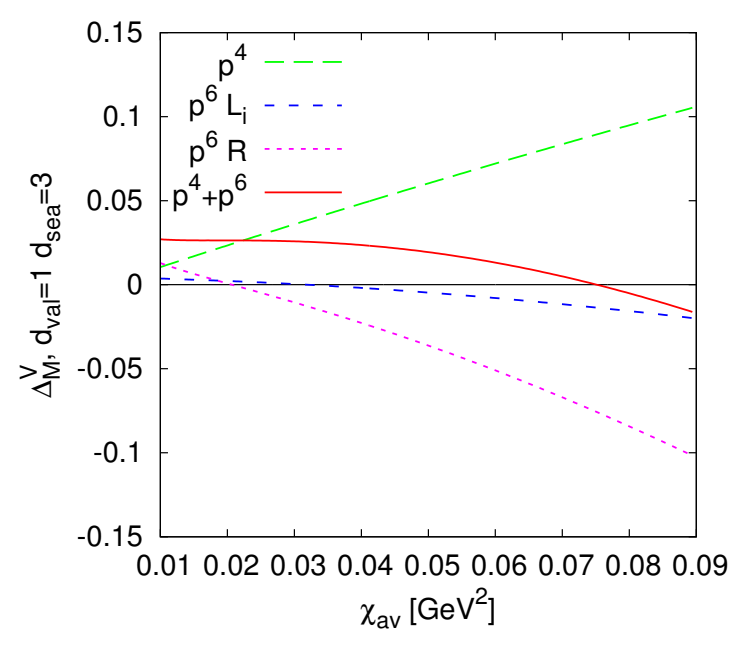

(c)

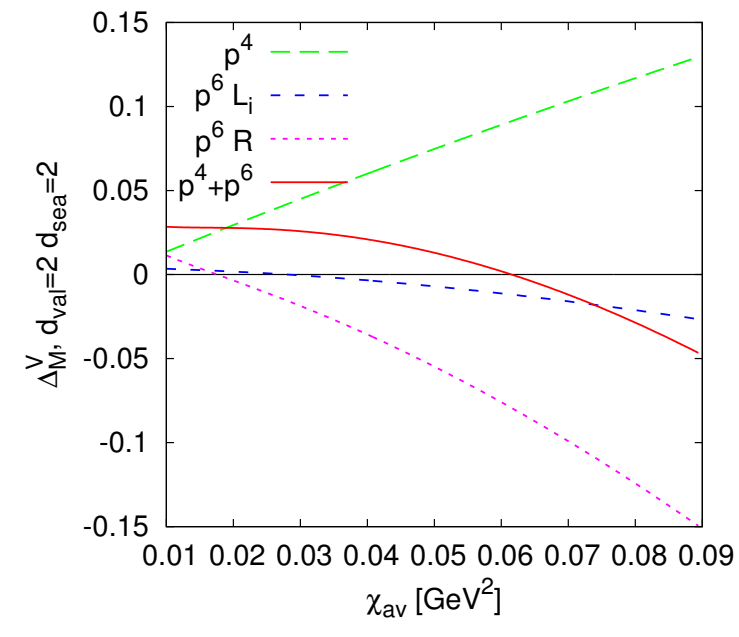

(b)

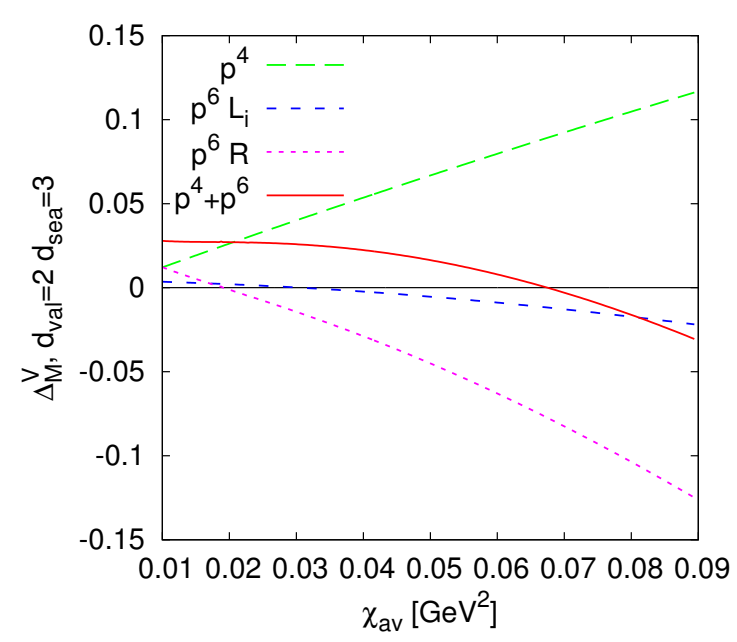

(d)

Figure 3. The corrections for the pion mass relative to the lowest order mass as a function of the average up and down sea quark mass via $\chi_{\mathrm{av}}$. (a) The isospin limit, $\chi_{1}=\chi_{2}, \chi_{4}=\chi_{5}=\chi_{\mathrm{av}}$. (b) Isospin breaking in the valence sector, $\chi_{1}=\chi_{3} / 2$ and $\chi_{4}=\chi_{5}=\chi_{\mathrm{av}}$. (c) Isospin breaking in the sea sector, $\chi_{1}=\chi_{2}$ and $\chi_{4}=\chi_{5} / 2$. (d) Isospin breaking in both sectors, $\chi_{1}=\chi_{3} / 2$ and $\chi_{4}=\chi_{5} / 2$.

in figure 3(a-d). We have checked numerically by using a different ratio for the isospin breaking that the corrections are indeed second order in isospin breaking.

\subsection{The pion decay constant}

In this subsection we look at the same cases as before. The lowest order mass is around the pion mass. We plot $\Delta_{F}^{V}$ with $\sqrt{\chi_{12}}=0.13 \mathrm{GeV}$, and as before $\sqrt{\chi_{6}}=$ $\sqrt{2(0.45)^{2}-(0.13)^{2}} \mathrm{GeV} \approx 0.623 \mathrm{GeV}$. The other input parameters are again chosen as given in the introduction of this section. We have restricted the sea up and down quark masses corresponding to a lowest order sea quark pion mass of 100 to $300 \mathrm{MeV}$. 


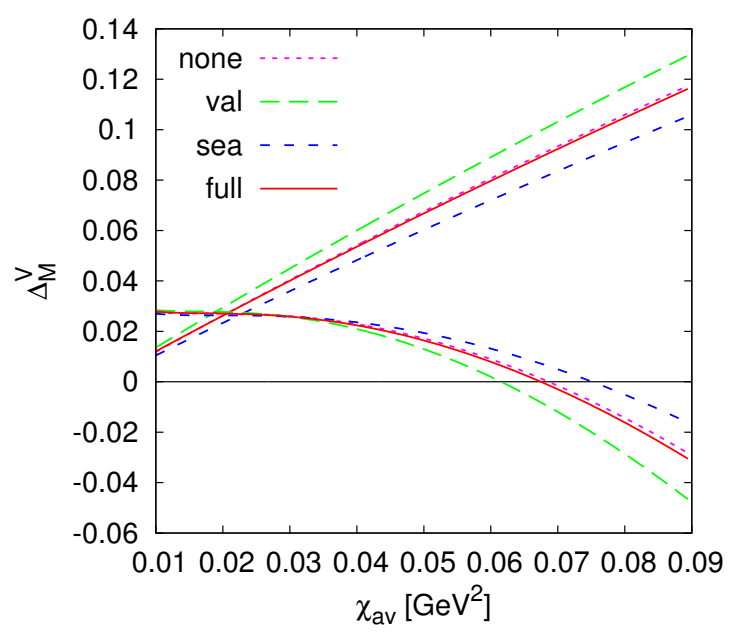

Figure 4. Comparing the finite volume correction for the meson masses for the cases with no isospin breaking (none), only in the valence sector (val), only in the sea sector (sea) and in both (full) for the meson mass squared. The upper curves are the $p^{4}$, the bottom the $p^{4}+p^{6}$ results.

The first case we look at is $d_{\text {val }}=1, d_{\text {sea }}=2$. This corresponds to taking the up and down quark masses equal in both the valence and sea quark sector and a different strange quark mass, i.e. the isospin limit. The result is shown in figure 5(a). The total $p^{6}$ correction is fairly small.

We now include isospin breaking in the valence sector. We thus look at the case with $d_{\text {val }}=2, d_{\text {sea }}=2$. We fix the valence quark masses such that $\chi_{1}+\chi_{2}=2 \chi_{12}$ and $\chi_{1} / \chi_{2}=1 / 2$. There is a sizable isospin breaking visible in the finite volume corrections, as shown in figure $3(\mathrm{~b})$.

The opposite case, isospin breaking in the sea sector but not in the valence sector leads to numerically much smaller effects. Here we used $\chi_{1}=\chi_{2}, \chi_{4}=\chi_{5} / 2$ and $\chi_{4}+\chi_{5}=2 \chi_{\mathrm{av}}$. The results are shown in figure $3(\mathrm{c})$.

Finally, we introduce isospin breaking in both the valence and sea quark sector with $\chi_{1} / \chi_{2}=1 / 2, \chi_{4}=\chi_{5} / 2$ and $\chi_{4}+\chi_{5}=2 \chi_{\mathrm{av}}$. The results are shown in figure $3(\mathrm{~d})$. The total isospin corrections are failry small.

The corrections due to valence and sea quark masses are all second order in isospin breaking. The same argument as in the unquenched case goes through both for the valence and sea quark masses. We compare the same four scenarios as for the pion mass, no isospin breaking, only in the valence sector, only in the sea sector and in both sectors. The curves are those shown in figure $5(\mathrm{a}-\mathrm{d})$. In figure 6 we compare the different isospin breaking cases for $p^{4}$ and $p^{4}+p^{6}$.

\subsection{The kaon mass and decay constant}

We now look only at the $d_{\text {val }}=d_{\text {sea }}=2$ case but choose the valence masses such that we have a lowest order pion mass of $130 \mathrm{MeV}$ and a lowest order kaon mass of $450 \mathrm{MeV}$. This corresponds to $\sqrt{\chi_{1}}=130 \mathrm{MeV}$ and $\sqrt{\chi_{3}} \approx 623 \mathrm{MeV}$. We plot the finite volume corrections 


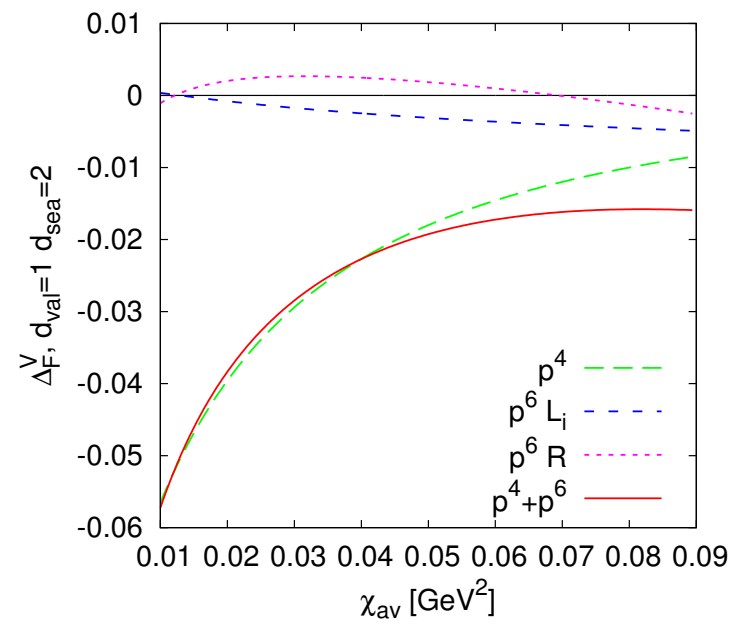

(a)

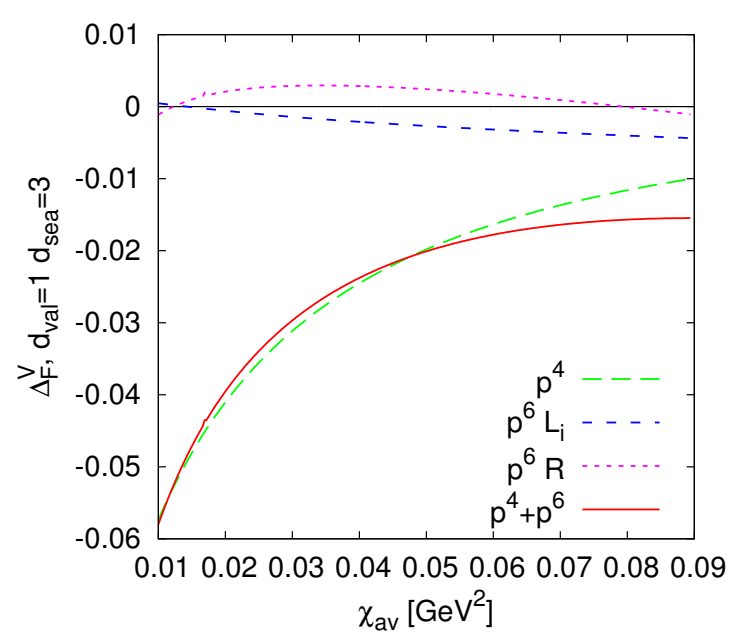

(c)

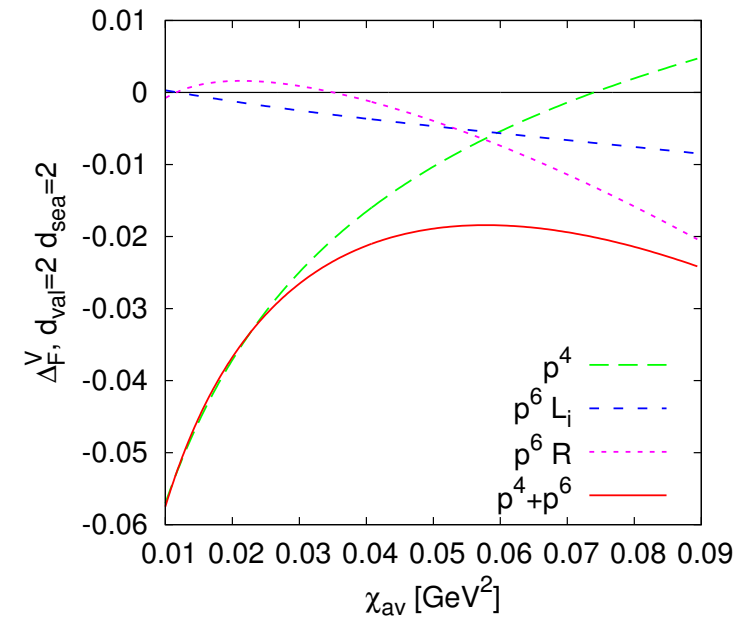

(b)

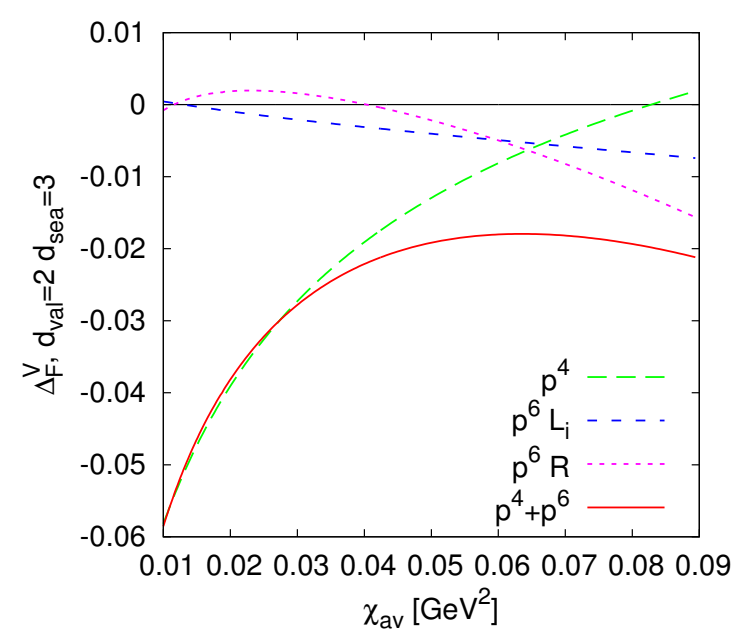

(d)

Figure 5. The corrections for the pion decay constant relative to its lowest order value as a function of the average up and down sea quark mass via $\chi_{\mathrm{av}}$. (a) The isospin limit, $\chi_{1}=\chi_{2}, \chi_{4}=\chi_{5}=\chi_{\mathrm{av}}$. (b) Isospin breaking in the valence sector, $\chi_{1}=\chi_{3} / 2$ and $\chi_{4}=\chi_{5}=\chi_{\mathrm{av}}$. (c) Isospin breaking in the sea sector, $\chi_{1}=\chi_{2}$ and $\chi_{4}=\chi_{5} / 2$. (d) Isospin breaking in both sectors, $\chi_{1}=\chi_{3} / 2$ and $\chi_{4}=\chi_{5} / 2$.

relative to the lowest order value of the quantity in figure 7 as a function of $\chi_{4}=\chi_{5}$. For the sea quark strange mass we use $\chi_{6}=1.02 \chi_{3}$. The LECs are again the ones from [38] and $L$ such that $M L=2$ for $M=130 \mathrm{MeV}$.

For the kaon we see that we reproduce the results of [15] that near the physical case the $p^{4}$ corrections are very small. The total finite volume corrections to the mass remain fairly small. The kaon decay constant has larger corrections but they remain in the few $\%$ region for the parameters considered. 


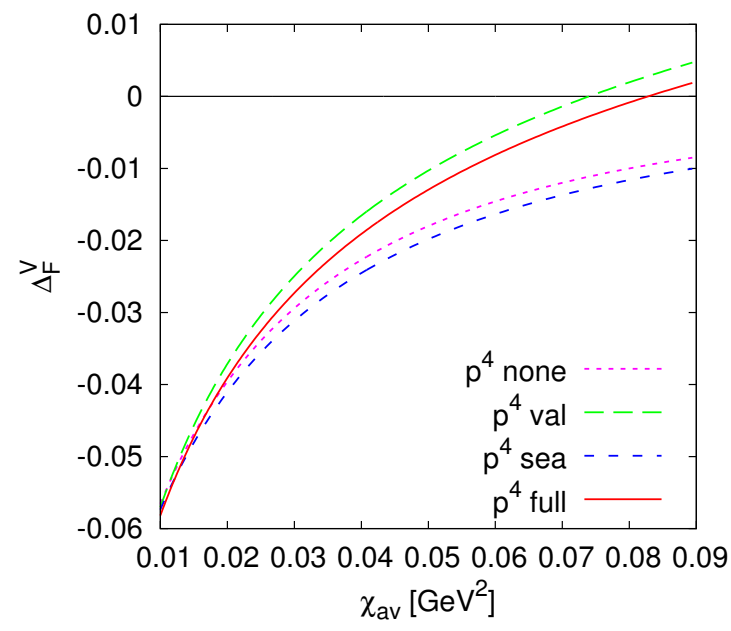

(a)

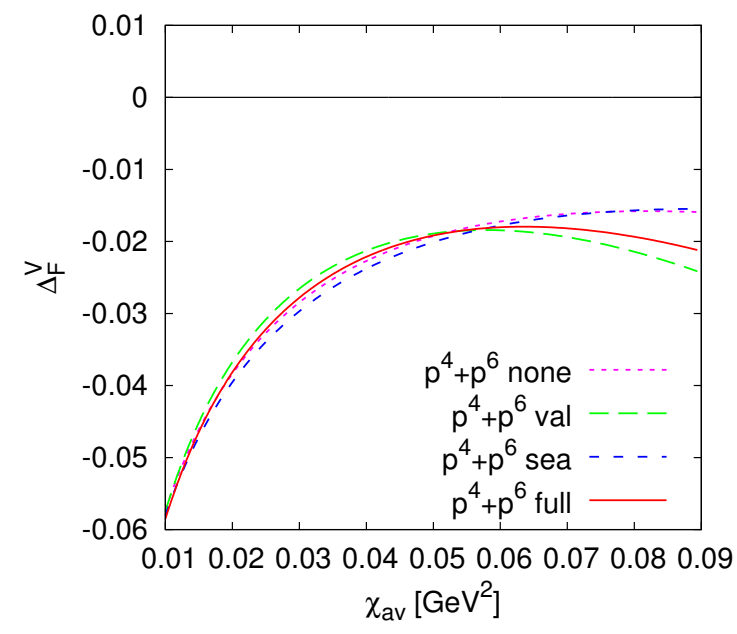

(b)

Figure 6. Comparing the finite volume correction for the meson decay constant and masses for the cases with no isospin breaking (none), only in the valence sector (val), only in the sea sector (sea) and in both (full) for the meson mass squared. (a) $p^{4}$ (b) $p^{4}+p^{6}$.

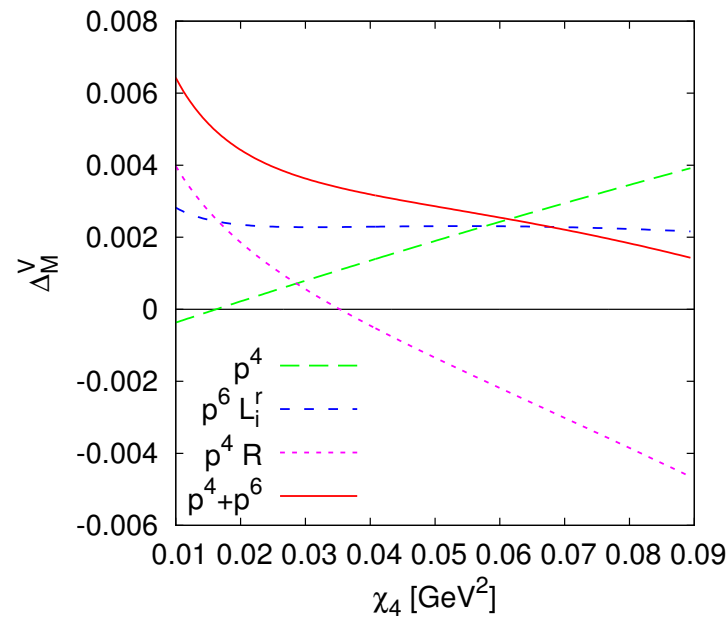

(a)

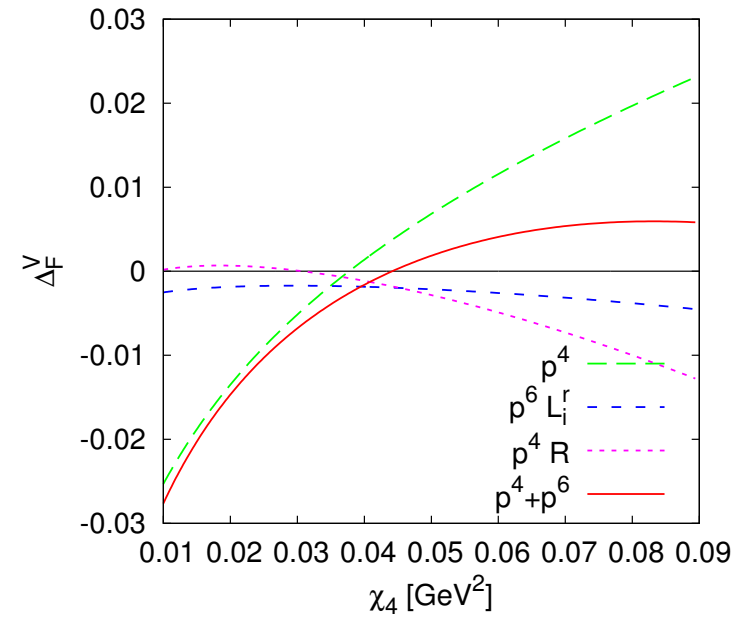

(b)

Figure 7. The finite volume corrections for a valence mass close to the kaon mass relative to the lowest order value. (a) the kaon mass squared. (b) the kaon decay constant.

\section{Conclusions}

We have computed the NNLO expressions for the masses and decay constants in threeflavour partially quenched ChPT for all possible mass cases. The calculation has been performed using two different formalisms, quark flow and the supersymmetric method. The known infinite volume expressions have been reproduced. We quoted the expressions for the case of equal valence and two different sea quark masses in the appendices. The other cases can be obtained from [22]. 
The numerical work shows finite volume corrections of a similar size as those in the unquenched case [15]. We have presented some representative numerics. The numerical work has been done using $\mathrm{C}++$. The programs are available together with the infinite volume results in [37]. The analytical work relied heavily on FORM [39].

\section{Acknowledgments}

This work is supported in part by the Swedish Research Council grants 621-2011-5080 and 621-2013-4287. JB thanks the Centro de Ciencias de Benasque Pedro Pascual, where part of this paper was written, for hospitality.

\section{A Expressions for the mass}

$F_{0}^{2} \Delta^{V} m_{12}^{2(4) 12}=+A^{V}\left(\chi_{1}\right)\left(-1 / 3 \chi_{1} R_{146 \eta}^{c}\right)+A^{V}\left(\chi_{\eta}\right)\left(2 / 3 \chi_{1} R_{\eta 61}^{z}{ }^{2}\right)+B^{V}\left(\chi_{1}\right)\left(-1 / 3 \chi_{1} R_{146 \eta}^{z}\right)$

$$
\begin{aligned}
& F_{0}^{4} \Delta^{V} m_{12}^{2(6 L) 12}=+A^{V}\left(\chi_{1}\right)\left(-64 / 3 \hat{L}_{8}^{r} \chi_{1}^{2} R_{146 \eta}^{c}-32 / 3 \hat{L}_{7}^{r} \chi_{1} \chi_{6} R_{14 \eta}^{z}-64 / 3 \hat{L}_{7}^{r} \chi_{1} \chi_{4} R_{16 \eta}^{z}\right. \\
& +32 \hat{L}_{7}^{r} \chi_{1}^{2}+32 \hat{L}_{6}^{r} \chi_{1}^{2}+8 / 3 \hat{L}_{5}^{r} \chi_{1} R_{146 \eta}^{z}-8 / 9 \hat{L}_{5}^{r} \chi_{1} \chi_{6} R_{14 \eta}^{z}{ }^{2}-16 / 9 \hat{L}_{5}^{r} \chi_{1} \chi_{4} R_{16 \eta}^{z}{ }^{2} \\
& +40 / 3 \hat{L}_{5}^{r} \chi_{1}^{2} R_{146 \eta}^{c}-8 \hat{L}_{3}^{r} \chi_{1} R_{146 \eta}^{z}-8 \hat{L}_{3}^{r} \chi_{1}^{2} R_{146 \eta}^{c}+20 \hat{L}_{2}^{r} \chi_{1}^{2}+8 \hat{L}_{1}^{r} \chi_{1}^{2}-8 \hat{L}_{0}^{r} \chi_{1} R_{146 \eta}^{z} \\
& -8 \hat{L}_{0}^{r} \chi_{1}^{2} R_{146 \eta}^{c}-16 / 3\left(\chi_{6}+2 \chi_{4}\right) \hat{L}_{6}^{r} \chi_{1} R_{146 \eta}^{c}-16 / 9\left(\chi_{6}+2 \chi_{4}\right) \hat{L}_{4}^{r} \chi_{1} R_{16 \eta}^{z}{ }^{2} \\
& \left.-8 / 9\left(\chi_{6}+2 \chi_{4}\right) \hat{L}_{4}^{r} \chi_{1} R_{14 \eta}^{z}{ }^{2}+8\left(\chi_{6}+2 \chi_{4}\right) \hat{L}_{4}^{r} \chi_{1} R_{146 \eta}^{c}\right)+A^{V}\left(\chi_{14}\right)\left(32\left(\chi_{4}+\chi_{1}\right) \hat{L}_{8}^{r} \chi_{1}\right. \\
& \left.-16\left(\chi_{4}+\chi_{1}\right) \hat{L}_{5}^{r} \chi_{1}+20\left(\chi_{4}+\chi_{1}\right) \hat{L}_{3}^{r} \chi_{1}+8\left(\chi_{4}+\chi_{1}\right) \hat{L}_{0}^{r} \chi_{1}\right)+A^{V}\left(\chi_{16}\right)\left(16\left(\chi_{6}+\chi_{1}\right) \hat{L}_{8}^{r} \chi_{1}\right. \\
& \left.-8\left(\chi_{6}+\chi_{1}\right) \hat{L}_{5}^{r} \chi_{1}+10\left(\chi_{6}+\chi_{1}\right) \hat{L}_{3}^{r} \chi_{1}+4\left(\chi_{6}+\chi_{1}\right) \hat{L}_{0}^{r} \chi_{1}\right)+A^{V}\left(\chi_{4}\right)\left(48 \hat{L}_{6}^{r} \chi_{1} \chi_{4}\right. \\
& \left.-48 \hat{L}_{4}^{r} \chi_{1} \chi_{4}+12 \hat{L}_{2}^{r} \chi_{1} \chi_{4}+48 \hat{L}_{1}^{r} \chi_{1} \chi_{4}\right)+A^{V}\left(\chi_{46}\right)\left(32\left(\chi_{6}+\chi_{4}\right) \hat{L}_{6}^{r} \chi_{1}-32\left(\chi_{6}+\chi_{4}\right) \hat{L}_{4}^{r} \chi_{1}\right. \\
& \left.+8\left(\chi_{6}+\chi_{4}\right) \hat{L}_{2}^{r} \chi_{1}+32\left(\chi_{6}+\chi_{4}\right) \hat{L}_{1}^{r} \chi_{1}\right)+A^{V}\left(\chi_{\eta}\right)\left(128 / 3 \hat{L}_{8}^{r} \chi_{1}^{2} R_{\eta 61}^{z}{ }^{2}\right. \\
& +32 / 9 \hat{L}_{5}^{r} \chi_{1} \chi_{6} R_{14 \eta}^{z} R_{\eta 61}^{z}-32 / 9 \hat{L}_{5}^{r} \chi_{1} \chi_{4} R_{16 \eta}^{z} R_{\eta 61}^{z}+16 \hat{L}_{3}^{r} \chi_{1} \chi_{\eta} R_{\eta 61}^{z}{ }^{2}+4 \hat{L}_{2}^{r} \chi_{1} \chi_{\eta} \\
& +16 \hat{L}_{1}^{r} \chi_{1} \chi_{\eta}+16 \hat{L}_{0}^{r} \chi_{1} \chi_{\eta} R_{\eta 61}^{z}{ }^{2}-8 / 3\left(3 \chi_{\eta}+2 \chi_{6}+\chi_{4}\right) \hat{L}_{4}^{r} \chi_{1} \\
& -16 / 9\left(3 \chi_{\eta}+2 \chi_{6}+\chi_{4}+12 \chi_{1}\right) \hat{L}_{5}^{r} \chi_{1} R_{\eta 61}^{z}{ }^{2}+64 / 3\left(\chi_{6}-\chi_{4}\right) \hat{L}_{7}^{r} \chi_{1} R_{\eta 61}^{z} \\
& +32 / 3\left(\chi_{6}+2 \chi_{4}\right) \hat{L}_{6}^{r} \chi_{1} R_{\eta 61}^{z}-64 / 3\left(\chi_{6}+2 \chi_{4}\right) \hat{L}_{4}^{r} \chi_{1} R_{\eta 61}^{z}{ }^{2} \\
& \left.-32 / 9\left(\chi_{6}+2 \chi_{4}\right) \hat{L}_{4}^{r} \chi_{1} R_{16 \eta}^{z} R_{\eta 61}^{z}+32 / 9\left(\chi_{6}+2 \chi_{4}\right) \hat{L}_{4}^{r} \chi_{1} R_{14 \eta}^{z} R_{\eta 61}^{z}+16 / 3\left(2 \chi_{6}+\chi_{4}\right) \hat{L}_{6}^{r} \chi_{1}\right) \\
& +B^{V}\left(\chi_{1}\right)\left(16 / 9 \hat{L}_{8}^{r} \chi_{1} \chi_{6}^{2} R_{14 \eta}^{z}+32 / 9 \hat{L}_{8}^{r} \chi_{1} \chi_{4}^{2} R_{16 \eta}^{z}{ }^{2}-64 / 3 \hat{L}_{8}^{r} \chi_{1}^{2} R_{146 \eta}^{z}-32 / 3 \hat{L}_{8}^{r} \chi_{1}^{3} R_{146 \eta}^{c}\right. \\
& +16 / 9 \hat{L}_{7}^{r} \chi_{1} \chi_{6}^{2} R_{14 \eta}^{z}{ }^{2}+64 / 9 \hat{L}_{7}^{r} \chi_{1} \chi_{4} \chi_{6} R_{14 \eta}^{z} R_{16 \eta}^{z}+64 / 9 \hat{L}_{7}^{r} \chi_{1} \chi_{4}^{2} R_{16 \eta}^{z}{ }^{2}-32 / 3 \hat{L}_{7}^{r} \chi_{1}^{2} \chi_{6} R_{14 \eta}^{z} \\
& -64 / 3 \hat{L}_{7}^{r} \chi_{1}^{2} \chi_{4} R_{16 \eta}^{z}+16 \hat{L}_{7}^{r} \chi_{1}^{3}+40 / 3 \hat{L}_{5}^{r} \chi_{1}^{2} R_{146 \eta}^{z}-8 / 9 \hat{L}_{5}^{r} \chi_{1}^{2} \chi_{6} R_{14 \eta}^{z}{ }^{2}-16 / 9 \hat{L}_{5}^{r} \chi_{1}^{2} \chi_{4} R_{16 \eta}^{z}{ }^{2} \\
& +16 / 3 \hat{L}_{5}^{r} \chi_{1}^{3} R_{146 \eta}^{c}-8 \hat{L}_{3}^{r} \chi_{1}^{2} R_{146 \eta}^{z}-8 \hat{L}_{0}^{r} \chi_{1}^{2} R_{146 \eta}^{z}-16 / 3\left(\chi_{6}+2 \chi_{4}\right) \hat{L}_{6}^{r} \chi_{1} R_{146 \eta}^{z} \\
& +16 / 9\left(\chi_{6}+2 \chi_{4}\right) \hat{L}_{6}^{r} \chi_{1} \chi_{6} R_{14 \eta}^{z}+32 / 9\left(\chi_{6}+2 \chi_{4}\right) \hat{L}_{6}^{r} \chi_{1} \chi_{4} R_{16 \eta}^{z}{ }^{2} \\
& -32 / 3\left(\chi_{6}+2 \chi_{4}\right) \hat{L}_{6}^{r} \chi_{1}^{2} R_{146 \eta}^{c}+8\left(\chi_{6}+2 \chi_{4}\right) \hat{L}_{4}^{r} \chi_{1} R_{146 \eta}^{z}-16 / 9\left(\chi_{6}+2 \chi_{4}\right) \hat{L}_{4}^{r} \chi_{1}^{2} R_{16 \eta}^{z}{ }^{2}
\end{aligned}
$$




$$
\begin{aligned}
& \left.-8 / 9\left(\chi_{6}+2 \chi_{4}\right) \hat{L}_{4}^{r} \chi_{1}^{2} R_{14 \eta}^{z}+16 / 3\left(\chi_{6}+2 \chi_{4}\right) \hat{L}_{4}^{r} \chi_{1}^{2} R_{146 \eta}^{c}\right) \\
& +B^{V}\left(\chi_{1}, \chi_{\eta}\right)\left(-64 / 9 \hat{L}_{8}^{r} \chi_{1} \chi_{6}^{2} R_{14 \eta}^{z} R_{\eta 61}^{z}+64 / 9 \hat{L}_{8}^{r} \chi_{1} \chi_{4}^{2} R_{16 \eta}^{z} R_{\eta 61}^{z}+64 / 3 \hat{L}_{8}^{r} \chi_{1}^{3} R_{\eta 61}^{z}{ }^{2}\right. \\
& +32 / 9 \hat{L}_{5}^{r} \chi_{1}^{2} \chi_{6} R_{14 \eta}^{z} R_{\eta 61}^{z}-32 / 9 \hat{L}_{5}^{r} \chi_{1}^{2} \chi_{4} R_{16 \eta}^{z} R_{\eta 61}^{z}-32 / 3 \hat{L}_{5}^{r} \chi_{1}^{3} R_{\eta 61}^{z}{ }^{2} \\
& -64 / 9\left(\chi_{6}-\chi_{4}\right) \hat{L}_{7}^{r} \chi_{1} \chi_{6} R_{14 \eta}^{z} R_{\eta 61}^{z}-128 / 9\left(\chi_{6}-\chi_{4}\right) \hat{L}_{7}^{r} \chi_{1} \chi_{4} R_{16 \eta}^{z} R_{\eta 61}^{z} \\
& +64 / 3\left(\chi_{6}-\chi_{4}\right) \hat{L}_{7}^{r} \chi_{1}^{2} R_{\eta 61}^{z}-64 / 9\left(\chi_{6}+2 \chi_{4}\right) \hat{L}_{6}^{r} \chi_{1} \chi_{6} R_{14 \eta}^{z} R_{\eta 61}^{z} \\
& +64 / 9\left(\chi_{6}+2 \chi_{4}\right) \hat{L}_{6}^{r} \chi_{1} \chi_{4} R_{16 \eta}^{z} R_{\eta 61}^{z}+64 / 3\left(\chi_{6}+2 \chi_{4}\right) \hat{L}_{6}^{r} \chi_{1}^{2} R_{\eta 61}^{z}{ }^{2} \\
& -32 / 3\left(\chi_{6}+2 \chi_{4}\right) \hat{L}_{4}^{r} \chi_{1}^{2} R_{\eta 61}^{z}-32 / 9\left(\chi_{6}+2 \chi_{4}\right) \hat{L}_{4}^{r} \chi_{1}^{2} R_{16 \eta}^{z} R_{\eta 61}^{z} \\
& \left.+32 / 9\left(\chi_{6}+2 \chi_{4}\right) \hat{L}_{4}^{r} \chi_{1}^{2} R_{14 \eta}^{z} R_{\eta 61}^{z}\right)+B^{V}\left(\chi_{\eta}\right)\left(64 / 9\left(\chi_{6}-\chi_{4}\right)^{2} \hat{L}_{7}^{r} \chi_{1} R_{\eta 61}^{z}{ }^{2}\right. \\
& -16 / 3\left(\chi_{6}+2 \chi_{4}\right) \hat{L}_{4}^{r} \chi_{1} \chi_{\eta} R_{\eta 61}^{z}+32 / 9\left(\chi_{6}+2 \chi_{4}\right)\left(2 \chi_{6}+\chi_{4}\right) \hat{L}_{6}^{r} \chi_{1} R_{\eta 61}^{z}{ }^{2} \\
& \left.-16 / 9\left(2 \chi_{6}+\chi_{4}\right) \hat{L}_{5}^{r} \chi_{1} \chi_{\eta} R_{\eta 61}^{z}+32 / 9\left(2 \chi_{6}^{2}+\chi_{4}^{2}\right) \hat{L}_{8}^{r} \chi_{1} R_{\eta 61}^{z}\right) \\
& +C^{V}\left(\chi_{1}\right)\left(-32 / 3 \hat{L}_{8}^{r} \chi_{1}^{3} R_{146 \eta}^{z}+16 / 3 \hat{L}_{5}^{r} \chi_{1}^{3} R_{146 \eta}^{z}-32 / 3\left(\chi_{6}+2 \chi_{4}\right) \hat{L}_{6}^{r} \chi_{1}^{2} R_{146 \eta}^{z}\right. \\
& \left.+16 / 3\left(\chi_{6}+2 \chi_{4}\right) \hat{L}_{4}^{r} \chi_{1}^{2} R_{146 \eta}^{z}\right)+A_{23}^{V}\left(\chi_{1}\right)\left(8 \hat{L}_{3}^{r} \chi_{1} R_{146 \eta}^{c}-12 \hat{L}_{2}^{r} \chi_{1}-24 \hat{L}_{1}^{r} \chi_{1}+8 \hat{L}_{0}^{r} \chi_{1} R_{146 \eta}^{c}\right) \\
& +A_{23}^{V}\left(\chi_{14}\right)\left(-24 \hat{L}_{3}^{r} \chi_{1}-48 \hat{L}_{0}^{r} \chi_{1}\right)+A_{23}^{V}\left(\chi_{16}\right)\left(-12 \hat{L}_{3}^{r} \chi_{1}-24 \hat{L}_{0}^{r} \chi_{1}\right) \\
& +A_{23}^{V}\left(\chi_{4}\right)\left(-36 \hat{L}_{2}^{r} \chi_{1}\right)+A_{23}^{V}\left(\chi_{46}\right)\left(-48 \hat{L}_{2}^{r} \chi_{1}\right)+A_{23}^{V}\left(\chi_{\eta}\right)\left(-16 \hat{L}_{3}^{r} \chi_{1} R_{\eta 61}^{z}-12 \hat{L}_{2}^{r} \chi_{1}\right. \\
& \left.-16 \hat{L}_{0}^{r} \chi_{1} R_{\eta 61}^{z}\right)+B_{23}^{V}\left(\chi_{1}\right)\left(8 \hat{L}_{3}^{r} \chi_{1} R_{146 \eta}^{z}+8 \hat{L}_{0}^{r} \chi_{1} R_{146 \eta}^{z}\right)
\end{aligned}
$$

$$
\begin{aligned}
& F_{0}^{4} \Delta^{V} m_{12}^{2(6 R) 12}=+\bar{A}\left(\chi_{1}\right) A^{V}\left(\chi_{1}\right)\left(\chi_{1}+1 / 9 \chi_{1} R_{146 \eta}^{c}{ }^{2}\right) \\
& +\bar{A}\left(\chi_{1}\right) A^{V}\left(\chi_{14}\right)\left(4 / 45 R_{146 \eta}^{z}+8 / 9 \chi_{1} R_{146 \eta}^{c}+4 / 45\left(\chi_{4}-11 \chi_{1}\right) R_{16 \eta}^{z}\right) \\
& +\bar{A}\left(\chi_{1}\right) A^{V}\left(\chi_{16}\right)\left(2 / 45 R_{146 \eta}^{z}+4 / 9 \chi_{1} R_{146 \eta}^{c}+2 / 45\left(\chi_{6}-11 \chi_{1}\right) R_{14 \eta}^{z}\right) \\
& +\bar{A}\left(\chi_{1}\right) A^{V}\left(\chi_{46}\right)\left(-2 / 27 \chi_{1} R_{16 \eta}^{z}{ }^{2}+4 / 27 \chi_{1} R_{14 \eta}^{z} R_{16 \eta}^{z}-2 / 27 \chi_{1} R_{14 \eta}^{z}{ }^{2}\right) \\
& +\bar{A}\left(\chi_{1}\right) A^{V}\left(\chi_{\eta}\right)\left(-2 / 9 \chi_{1} R_{146 \eta}^{c} R_{\eta 61}^{z}\right)+\bar{A}\left(\chi_{1}\right) B^{V}\left(\chi_{1}\right)\left(1 / 9 \chi_{1} R_{146 \eta}^{z} R_{146 \eta}^{c}\right. \\
& \left.+\chi_{1}^{2}+2 / 9 \chi_{1}^{2} R_{146 \eta}^{c}{ }^{2}\right)+\bar{A}\left(\chi_{1}\right) B^{V}\left(\chi_{1}, \chi_{\eta}\right)\left(-8 / 9 \chi_{1}^{2} R_{146 \eta}^{c} R_{\eta 61}^{z}{ }^{2}\right) \\
& +\bar{A}\left(\chi_{1}\right) C^{V}\left(\chi_{1}\right)\left(2 / 9 \chi_{1}^{2} R_{146 \eta}^{z} R_{146 \eta}^{c}\right)+\bar{A}\left(\chi_{14}\right) A^{V}\left(\chi_{1}\right)\left(4 / 45 R_{146 \eta}^{z}+8 / 9 \chi_{1} R_{146 \eta}^{c}\right. \\
& \left.+4 / 45\left(\chi_{4}-11 \chi_{1}\right) R_{16 \eta}^{z}\right)+\bar{A}\left(\chi_{14}\right) A^{V}\left(\chi_{14}\right)\left(-4 / 27 R_{146 \eta}^{z}+10 / 27 \chi_{1} R_{16 \eta}^{z}\right. \\
& +1 / 27\left(2 \chi_{\eta}-2 \chi_{4}-47 \chi_{1}\right)+2 / 27\left(4 \chi_{\eta}+4 \chi_{4}+9 \chi_{1}\right) R_{\eta 61}^{z}+2 / 27\left(4 \chi_{\eta}+\chi_{1}\right) R_{\eta 61}^{z} \\
& \left.-1 / 27\left(4 \chi_{4}+13 \chi_{1}\right) R_{146 \eta}^{c}\right)+\bar{A}\left(\chi_{14}\right) A^{V}\left(\chi_{16}\right)\left(-\chi_{1}\right)+\bar{A}\left(\chi_{14}\right) A^{V}\left(\chi_{\eta}\right)\left(-2 / 45\left(\chi_{\eta}-\chi_{4}\right)\right. \\
& \left.-8 / 45\left(\chi_{\eta}+9 \chi_{1}\right) R_{\eta 61}^{z}{ }^{2}-4 / 45\left(2 \chi_{\eta}-\chi_{4}+9 \chi_{1}\right) R_{\eta 61}^{z}\right)+\bar{A}\left(\chi_{14}\right) B^{V}\left(\chi_{1}\right)\left(8 / 9 \chi_{1} R_{146 \eta}^{z}\right. \\
& \left.-4 / 9\left(\chi_{4}+2 \chi_{1}\right) \chi_{1} R_{16 \eta}^{z}\right)+\bar{A}\left(\chi_{14}\right) B^{V}\left(\chi_{1}, \chi_{\eta}\right)\left(-4 / 9\left(\chi_{4}+2 \chi_{1}\right) \chi_{1} R_{\eta 61}^{z}\right) \\
& +\bar{A}\left(\chi_{16}\right) A^{V}\left(\chi_{1}\right)\left(2 / 45 R_{146 \eta}^{z}+4 / 9 \chi_{1} R_{146 \eta}^{c}+2 / 45\left(\chi_{6}-11 \chi_{1}\right) R_{14 \eta}^{z}\right)
\end{aligned}
$$




$$
\begin{aligned}
& +\bar{A}\left(\chi_{16}\right) A^{V}\left(\chi_{14}\right)\left(-\chi_{1}\right)+\bar{A}\left(\chi_{16}\right) A^{V}\left(\chi_{16}\right)\left(-2 / 27 R_{146 \eta}^{z}+5 / 27 \chi_{1} R_{14 \eta}^{z}\right. \\
& +2 / 27\left(2 \chi_{\eta}-2 \chi_{6}-5 \chi_{1}\right)+1 / 27\left(4 \chi_{\eta}+4 \chi_{6}+9 \chi_{1}\right) R_{\eta 61}^{z}{ }^{2}-2 / 27\left(4 \chi_{\eta}+\chi_{1}\right) R_{\eta 61}^{z} \\
& \left.-1 / 54\left(4 \chi_{6}+13 \chi_{1}\right) R_{146 \eta}^{c}\right)+\bar{A}\left(\chi_{16}\right) A^{V}\left(\chi_{\eta}\right)\left(-4 / 45\left(\chi_{\eta}-\chi_{6}\right)\right. \\
& \left.-4 / 45\left(\chi_{\eta}+9 \chi_{1}\right) R_{\eta 61}^{z}{ }^{2}+4 / 45\left(2 \chi_{\eta}-\chi_{6}+9 \chi_{1}\right) R_{\eta 61}^{z}\right)+\bar{A}\left(\chi_{16}\right) B^{V}\left(\chi_{1}\right)\left(4 / 9 \chi_{1} R_{146 \eta}^{z}\right. \\
& \left.-2 / 9\left(\chi_{6}+2 \chi_{1}\right) \chi_{1} R_{14 \eta}^{z}\right)+\bar{A}\left(\chi_{16}\right) B^{V}\left(\chi_{1}, \chi_{\eta}\right)\left(4 / 9\left(\chi_{6}+2 \chi_{1}\right) \chi_{1} R_{\eta 61}^{z}\right) \\
& +\bar{A}\left(\chi_{4}\right) B^{V}\left(\chi_{1}\right)\left(1 / 3 \chi_{1} \chi_{4} R_{16 \eta}^{z}{ }^{2}\right)+\bar{A}\left(\chi_{4}\right) B^{V}\left(\chi_{1}, \chi_{\eta}\right)\left(2 / 3 \chi_{1} \chi_{4} R_{16 \eta}^{z} R_{\eta 61}^{z}\right) \\
& +\bar{A}\left(\chi_{4}\right) B^{V}\left(\chi_{\eta}\right)\left(1 / 3 \chi_{1} \chi_{4} R_{\eta 61}^{z}{ }^{2}\right)+\bar{A}\left(\chi_{46}\right) A^{V}\left(\chi_{1}\right)\left(-2 / 27 \chi_{1} R_{16 \eta}^{z}{ }^{2}+4 / 27 \chi_{1} R_{14 \eta}^{z} R_{16 \eta}^{z}\right. \\
& \left.-2 / 27 \chi_{1} R_{14 \eta}^{z}{ }^{2}\right)+\bar{A}\left(\chi_{46}\right) A^{V}\left(\chi_{\eta}\right)\left(-2 / 3 \chi_{1} R_{\eta 61}^{z}{ }^{2}-4 / 9 \chi_{1} R_{16 \eta}^{z} R_{\eta 61}^{z}+4 / 9 \chi_{1} R_{14 \eta}^{z} R_{\eta 61}^{z}\right) \\
& +\bar{A}\left(\chi_{46}\right) B^{V}\left(\chi_{1}\right)\left(4 / 27\left(\chi_{6}+\chi_{4}+\chi_{1}\right) \chi_{1} R_{14 \eta}^{z} R_{16 \eta}^{z}+2 / 27\left(\chi_{6}-\chi_{1}\right) \chi_{1} R_{14 \eta}^{z}{ }^{2}\right. \\
& \left.+2 / 27\left(\chi_{4}-\chi_{1}\right) \chi_{1} R_{16 \eta}^{z}{ }^{2}\right)+\bar{A}\left(\chi_{46}\right) B^{V}\left(\chi_{1}, \chi_{\eta}\right)\left(-4 / 27\left(\chi_{6}-\chi_{4}-3 \chi_{1}\right) \chi_{1} R_{14 \eta}^{z} R_{\eta 61}^{z}\right. \\
& \left.-4 / 27\left(2 \chi_{6}+\chi_{4}+3 \chi_{1}\right) \chi_{1} R_{16 \eta}^{z} R_{\eta 61}^{z}\right)+\bar{A}\left(\chi_{46}\right) B^{V}\left(\chi_{\eta}\right)\left(-2 / 9\left(3 \chi_{\eta}+\chi_{4}\right) \chi_{1} R_{\eta 61}^{z}{ }^{2}\right) \\
& +\bar{A}\left(\chi_{\eta}\right) A^{V}\left(\chi_{1}\right)\left(-2 / 9 \chi_{1} R_{146 \eta}^{c} R_{\eta 61}^{z}\right)+\bar{A}\left(\chi_{\eta}\right) A^{V}\left(\chi_{14}\right)\left(-2 / 45\left(\chi_{\eta}-\chi_{4}\right)\right. \\
& \left.-8 / 45\left(\chi_{\eta}+9 \chi_{1}\right) R_{\eta 61}^{z}{ }^{2}-4 / 45\left(2 \chi_{\eta}-\chi_{4}+9 \chi_{1}\right) R_{\eta 61}^{z}\right)+\bar{A}\left(\chi_{\eta}\right) A^{V}\left(\chi_{16}\right)\left(-4 / 45\left(\chi_{\eta}-\chi_{6}\right)\right. \\
& \left.-4 / 45\left(\chi_{\eta}+9 \chi_{1}\right) R_{\eta 61}^{z}{ }^{2}+4 / 45\left(2 \chi_{\eta}-\chi_{6}+9 \chi_{1}\right) R_{\eta 61}^{z}\right)+\bar{A}\left(\chi_{\eta}\right) A^{V}\left(\chi_{46}\right)\left(-2 / 3 \chi_{1} R_{\eta 61}^{z}{ }^{2}\right. \\
& \left.-4 / 9 \chi_{1} R_{16 \eta}^{z} R_{\eta 61}^{z}+4 / 9 \chi_{1} R_{14 \eta}^{z} R_{\eta 61}^{z}\right)+\bar{A}\left(\chi_{\eta}\right) A^{V}\left(\chi_{\eta}\right)\left(4 / 9 \chi_{1} R_{\eta 61}^{z}{ }^{4}\right) \\
& +\bar{A}\left(\chi_{\eta}\right) B^{V}\left(\chi_{1}\right)\left(-2 / 9 \chi_{1} R_{146 \eta}^{z} R_{\eta 61}^{z}{ }^{2}+2 / 27 \chi_{1} \chi_{6} R_{14 \eta}^{z}{ }^{2}+1 / 27 \chi_{1} \chi_{4} R_{16 \eta}^{z}{ }^{2}\right. \\
& \left.-4 / 9 \chi_{1}^{2} R_{146 \eta}^{c} R_{\eta 61}^{z}\right)+\bar{A}\left(\chi_{\eta}\right) B^{V}\left(\chi_{1}, \chi_{\eta}\right)\left(-8 / 27 \chi_{1} \chi_{6} R_{14 \eta}^{z} R_{\eta 61}^{z}+2 / 27 \chi_{1} \chi_{4} R_{16 \eta}^{z} R_{\eta 61}^{z}\right. \\
& \left.+8 / 9 \chi_{1}^{2} R_{\eta 61}^{z}{ }^{4}+4 / 9 \chi_{1}^{2} R_{146 \eta}^{c} R_{\eta 61}^{z}{ }^{2}\right)+\bar{A}\left(\chi_{\eta}\right) B^{V}\left(\chi_{\eta}\right)\left(1 / 27\left(8 \chi_{6}+\chi_{4}\right) \chi_{1} R_{\eta 61}^{z}{ }^{2}\right) \\
& +\bar{A}\left(\chi_{\eta}\right) C^{V}\left(\chi_{1}\right)\left(-4 / 9 \chi_{1}^{2} R_{146 \eta}^{z} R_{\eta 61}^{z}{ }^{2}\right)+\bar{B}\left(\chi_{1}\right) A^{V}\left(\chi_{1}\right)\left(1 / 9 \chi_{1} R_{146 \eta}^{z} R_{146 \eta}^{c}+\chi_{1}^{2}\right. \\
& \left.+2 / 9 \chi_{1}^{2} R_{146 \eta}^{c}{ }^{2}\right)+\bar{B}\left(\chi_{1}\right) A^{V}\left(\chi_{14}\right)\left(8 / 9 \chi_{1} R_{146 \eta}^{z}-4 / 9\left(\chi_{4}+2 \chi_{1}\right) \chi_{1} R_{16 \eta}^{z}\right) \\
& +\bar{B}\left(\chi_{1}\right) A^{V}\left(\chi_{16}\right)\left(4 / 9 \chi_{1} R_{146 \eta}^{z}-2 / 9\left(\chi_{6}+2 \chi_{1}\right) \chi_{1} R_{14 \eta}^{z}\right)+\bar{B}\left(\chi_{1}\right) A^{V}\left(\chi_{4}\right)\left(1 / 3 \chi_{1} \chi_{4} R_{16 \eta}^{z}{ }^{2}\right) \\
& +\bar{B}\left(\chi_{1}\right) A^{V}\left(\chi_{46}\right)\left(4 / 27\left(\chi_{6}+\chi_{4}+\chi_{1}\right) \chi_{1} R_{14 \eta}^{z} R_{16 \eta}^{z}+2 / 27\left(\chi_{6}-\chi_{1}\right) \chi_{1} R_{14 \eta}^{z}{ }^{2}\right. \\
& \left.+2 / 27\left(\chi_{4}-\chi_{1}\right) \chi_{1} R_{16 \eta}^{z}{ }^{2}\right)+\bar{B}\left(\chi_{1}\right) A^{V}\left(\chi_{\eta}\right)\left(-2 / 9 \chi_{1} R_{146 \eta}^{z} R_{\eta 61}^{z}+2 / 27 \chi_{1} \chi_{6} R_{14 \eta}^{z}{ }^{2}\right. \\
& \left.+1 / 27 \chi_{1} \chi_{4} R_{16 \eta}^{z}{ }^{2}-4 / 9 \chi_{1}^{2} R_{146 \eta}^{c} R_{\eta 61}^{z}{ }^{2}\right)+\bar{B}\left(\chi_{1}\right) B^{V}\left(\chi_{1}\right)\left(1 / 9 \chi_{1} R_{146 \eta}^{z}{ }^{2}+4 / 9 \chi_{1}^{2} R_{146 \eta}^{z} R_{146 \eta}^{c}\right) \\
& +\bar{B}\left(\chi_{1}\right) B^{V}\left(\chi_{1}, \chi_{\eta}\right)\left(-4 / 9 \chi_{1}^{2} R_{146 \eta}^{z} R_{\eta 61}^{z}{ }^{2}\right)+\bar{B}\left(\chi_{1}\right) C^{V}\left(\chi_{1}\right)\left(2 / 9 \chi_{1}^{2} R_{146 \eta}^{z}{ }^{2}\right) \\
& +\bar{B}\left(\chi_{1}, \chi_{\eta}\right) A^{V}\left(\chi_{14}\right)\left(-4 / 9\left(\chi_{4}+2 \chi_{1}\right) \chi_{1} R_{\eta 61}^{z}\right)+\bar{B}\left(\chi_{1}, \chi_{\eta}\right) A^{V}\left(\chi_{16}\right)\left(4 / 9\left(\chi_{6}\right.\right. \\
& \left.\left.+2 \chi_{1}\right) \chi_{1} R_{\eta 61}^{z}\right)+\bar{B}\left(\chi_{1}, \chi_{\eta}\right) A^{V}\left(\chi_{4}\right)\left(2 / 3 \chi_{1} \chi_{4} R_{16 \eta}^{z} R_{\eta 61}^{z}\right)
\end{aligned}
$$




$$
\begin{aligned}
& +\bar{B}\left(\chi_{1}, \chi_{\eta}\right) A^{V}\left(\chi_{46}\right)\left(-4 / 27\left(\chi_{6}-\chi_{4}-3 \chi_{1}\right) \chi_{1} R_{14 \eta}^{z} R_{\eta 61}^{z}-4 / 27\left(2 \chi_{6}+\chi_{4}\right.\right. \\
& \left.\left.+3 \chi_{1}\right) \chi_{1} R_{16 \eta}^{z} R_{\eta 61}^{z}\right)+\bar{B}\left(\chi_{1}, \chi_{\eta}\right) A^{V}\left(\chi_{\eta}\right)\left(-8 / 27 \chi_{1} \chi_{6} R_{14 \eta}^{z} R_{\eta 61}^{z}+2 / 27 \chi_{1} \chi_{4} R_{16 \eta}^{z} R_{\eta 61}^{z}\right. \\
& \left.+8 / 9 \chi_{1}^{2} R_{\eta 61}^{z}{ }^{4}-4 / 9 \chi_{1}^{2} R_{146 \eta}^{c} R_{\eta 61}^{z}{ }^{2}\right)+\bar{B}\left(\chi_{1}, \chi_{\eta}\right) B^{V}\left(\chi_{1}\right)\left(-4 / 9 \chi_{1}^{2} R_{146 \eta}^{z} R_{\eta 61}^{z}{ }^{2}\right) \\
& +\bar{B}\left(\chi_{\eta}\right) A^{V}\left(\chi_{4}\right)\left(1 / 3 \chi_{1} \chi_{4} R_{\eta 61}^{z}{ }^{2}\right)+\bar{B}\left(\chi_{\eta}\right) A^{V}\left(\chi_{46}\right)\left(-2 / 9\left(3 \chi_{\eta}+\chi_{4}\right) \chi_{1} R_{\eta 61}^{z}{ }^{2}\right) \\
& +\bar{B}\left(\chi_{\eta}\right) A^{V}\left(\chi_{\eta}\right)\left(1 / 27\left(8 \chi_{6}+\chi_{4}\right) \chi_{1} R_{\eta 61}^{z}{ }^{2}\right)+\bar{C}\left(\chi_{1}\right) A^{V}\left(\chi_{1}\right)\left(2 / 9 \chi_{1}^{2} R_{146 \eta}^{z} R_{146 \eta}^{c}\right) \\
& +\bar{C}\left(\chi_{1}\right) A^{V}\left(\chi_{\eta}\right)\left(-4 / 9 \chi_{1}^{2} R_{146 \eta}^{z} R_{\eta 61}^{z}{ }^{2}\right)+\bar{C}\left(\chi_{1}\right) B^{V}\left(\chi_{1}\right)\left(2 / 9 \chi_{1}^{2} R_{146 \eta}^{z}{ }^{2}\right) \\
& +A^{V}\left(\chi_{1}\right) \frac{1}{16 \pi^{2}}\left(-1 / 4 \chi_{1}^{2}+1 / 6 \chi_{1}^{2} R_{16 \eta}^{z}+1 / 12 \chi_{1}^{2} R_{14 \eta}^{z}\right)+A^{V}\left(\chi_{1}\right)^{2}\left(1 / 2 \chi_{1}+1 / 18 \chi_{1} R_{146 \eta}^{c}{ }^{2}\right) \\
& +A^{V}\left(\chi_{1}\right) A^{V}\left(\chi_{14}\right)\left(4 / 45 R_{146 \eta}^{z}+8 / 9 \chi_{1} R_{146 \eta}^{c}+4 / 45\left(\chi_{4}-11 \chi_{1}\right) R_{16 \eta}^{z}\right) \\
& +A^{V}\left(\chi_{1}\right) A^{V}\left(\chi_{16}\right)\left(2 / 45 R_{146 \eta}^{z}+4 / 9 \chi_{1} R_{146 \eta}^{c}+2 / 45\left(\chi_{6}-11 \chi_{1}\right) R_{14 \eta}^{z}\right) \\
& +A^{V}\left(\chi_{1}\right) A^{V}\left(\chi_{46}\right)\left(-2 / 27 \chi_{1} R_{16 \eta}^{z}{ }^{2}+4 / 27 \chi_{1} R_{14 \eta}^{z} R_{16 \eta}^{z}-2 / 27 \chi_{1} R_{14 \eta}^{z}{ }^{2}\right) \\
& +A^{V}\left(\chi_{1}\right) A^{V}\left(\chi_{\eta}\right)\left(-2 / 9 \chi_{1} R_{146 \eta}^{c} R_{\eta 61}^{z}{ }^{2}\right)+A^{V}\left(\chi_{1}\right) B^{V}\left(\chi_{1}\right)\left(1 / 9 \chi_{1} R_{146 \eta}^{z} R_{146 \eta}^{c}+\chi_{1}^{2}\right. \\
& \left.+2 / 9 \chi_{1}^{2} R_{146 \eta}^{c}{ }^{2}\right)+A^{V}\left(\chi_{1}\right) B^{V}\left(\chi_{1}, \chi_{\eta}\right)\left(-4 / 9 \chi_{1}^{2} R_{146 \eta}^{c} R_{\eta 61}^{z}{ }^{2}\right) \\
& +A^{V}\left(\chi_{1}\right) C^{V}\left(\chi_{1}\right)\left(2 / 9 \chi_{1}^{2} R_{146 \eta}^{z} R_{146 \eta}^{c}\right)+A^{V}\left(\chi_{14}\right) \frac{1}{16 \pi^{2}}\left(-1 / 6 \chi_{1} R_{146 \eta}^{z}+1 / 36\left(3 \chi_{\eta}+18 \chi_{6}\right.\right. \\
& \left.+42 \chi_{4}-\chi_{1}\right) \chi_{1}+1 / 18\left(6 \chi_{\eta}+3 \chi_{4}+\chi_{1}\right) \chi_{1} R_{\eta 61}^{z}+1 / 18\left(6 \chi_{\eta}+3 \chi_{4}+\chi_{1}\right) \chi_{1} R_{\eta 61}^{z}{ }^{2} \\
& \left.+1 / 18\left(3 \chi_{4}+7 \chi_{1}\right) \chi_{1} R_{16 \eta}^{z}-1 / 36\left(3 \chi_{4}+7 \chi_{1}\right) \chi_{1} R_{146 \eta}^{c}\right)+A^{V}\left(\chi_{14}\right)^{2}\left(-2 / 27 R_{146 \eta}^{z}\right. \\
& +5 / 27 \chi_{1} R_{16 \eta}^{z}+1 / 54\left(2 \chi_{\eta}-2 \chi_{4}-47 \chi_{1}\right)+1 / 27\left(4 \chi_{\eta}+4 \chi_{4}+9 \chi_{1}\right) R_{\eta 61}^{z}{ }^{2} \\
& \left.+1 / 27\left(4 \chi_{\eta}+\chi_{1}\right) R_{\eta 61}^{z}-1 / 54\left(4 \chi_{4}+13 \chi_{1}\right) R_{146 \eta}^{c}\right)+A^{V}\left(\chi_{14}\right) A^{V}\left(\chi_{16}\right)\left(-\chi_{1}\right) \\
& +A^{V}\left(\chi_{14}\right) A^{V}\left(\chi_{\eta}\right)\left(-2 / 45\left(\chi_{\eta}-\chi_{4}\right)-8 / 45\left(\chi_{\eta}+9 \chi_{1}\right) R_{\eta 61}^{z}{ }^{2}-4 / 45\left(2 \chi_{\eta}-\chi_{4}+9 \chi_{1}\right) R_{\eta 61}^{z}\right) \\
& +A^{V}\left(\chi_{14}\right) B^{V}\left(\chi_{1}\right)\left(8 / 9 \chi_{1} R_{146 \eta}^{z}-4 / 9\left(\chi_{4}+2 \chi_{1}\right) \chi_{1} R_{16 \eta}^{z}\right) \\
& +A^{V}\left(\chi_{14}\right) B^{V}\left(\chi_{1}, \chi_{\eta}\right)\left(-4 / 9\left(\chi_{4}+2 \chi_{1}\right) \chi_{1} R_{\eta 61}^{z}\right)+A^{V}\left(\chi_{16}\right) \frac{1}{16 \pi^{2}}\left(-1 / 12 \chi_{1} R_{146 \eta}^{z}\right. \\
& -1 / 18\left(6 \chi_{\eta}+3 \chi_{6}+\chi_{1}\right) \chi_{1} R_{\eta 61}^{z}+1 / 36\left(6 \chi_{\eta}+3 \chi_{6}+\chi_{1}\right) \chi_{1} R_{\eta 61}^{z}+1 / 72\left(12 \chi_{\eta}+15 \chi_{6}\right. \\
& \left.\left.+36 \chi_{4}-\chi_{1}\right) \chi_{1}+1 / 36\left(3 \chi_{6}+7 \chi_{1}\right) \chi_{1} R_{14 \eta}^{z}-1 / 72\left(3 \chi_{6}+7 \chi_{1}\right) \chi_{1} R_{146 \eta}^{c}\right) \\
& +A^{V}\left(\chi_{16}\right)^{2}\left(-1 / 27 R_{146 \eta}^{z}+5 / 54 \chi_{1} R_{14 \eta}^{z}+1 / 27\left(2 \chi_{\eta}-2 \chi_{6}-5 \chi_{1}\right)+1 / 54\left(4 \chi_{\eta}+4 \chi_{6}\right.\right. \\
& \left.\left.+9 \chi_{1}\right) R_{\eta 61}^{z}{ }^{2}-1 / 27\left(4 \chi_{\eta}+\chi_{1}\right) R_{\eta 61}^{z}-1 / 108\left(4 \chi_{6}+13 \chi_{1}\right) R_{146 \eta}^{c}\right) \\
& +A^{V}\left(\chi_{16}\right) A^{V}\left(\chi_{\eta}\right)\left(-4 / 45\left(\chi_{\eta}-\chi_{6}\right)-4 / 45\left(\chi_{\eta}+9 \chi_{1}\right) R_{\eta 61}^{z}{ }^{2}+4 / 45\left(2 \chi_{\eta}-\chi_{6}+9 \chi_{1}\right) R_{\eta 61}^{z}\right) \\
& +A^{V}\left(\chi_{16}\right) B^{V}\left(\chi_{1}\right)\left(4 / 9 \chi_{1} R_{146 \eta}^{z}-2 / 9\left(\chi_{6}+2 \chi_{1}\right) \chi_{1} R_{14 \eta}^{z}\right)+A^{V}\left(\chi_{16}\right) B^{V}\left(\chi_{1}, \chi_{\eta}\right)\left(4 / 9\left(\chi_{6}\right.\right. \\
& \left.\left.+2 \chi_{1}\right) \chi_{1} R_{\eta 61}^{z}\right)+A^{V}\left(\chi_{4}\right) B^{V}\left(\chi_{1}\right)\left(1 / 3 \chi_{1} \chi_{4} R_{16 \eta}^{z}{ }^{2}\right)
\end{aligned}
$$


$+A^{V}\left(\chi_{4}\right) B^{V}\left(\chi_{1}, \chi_{\eta}\right)\left(2 / 3 \chi_{1} \chi_{4} R_{16 \eta}^{z} R_{\eta 61}^{z}\right)+A^{V}\left(\chi_{4}\right) B^{V}\left(\chi_{\eta}\right)\left(1 / 3 \chi_{1} \chi_{4} R_{\eta 61}^{z}{ }^{2}\right)$

$+A^{V}\left(\chi_{46}\right) A^{V}\left(\chi_{\eta}\right)\left(-2 / 3 \chi_{1} R_{\eta 61}^{z}{ }^{2}-4 / 9 \chi_{1} R_{16 \eta}^{z} R_{\eta 61}^{z}+4 / 9 \chi_{1} R_{14 \eta}^{z} R_{\eta 61}^{z}\right)$

$+A^{V}\left(\chi_{46}\right) B^{V}\left(\chi_{1}\right)\left(4 / 27\left(\chi_{6}+\chi_{4}+\chi_{1}\right) \chi_{1} R_{14 \eta}^{z} R_{16 \eta}^{z}+2 / 27\left(\chi_{6}-\chi_{1}\right) \chi_{1} R_{14 \eta}^{z}{ }^{2}\right.$

$\left.+2 / 27\left(\chi_{4}-\chi_{1}\right) \chi_{1} R_{16 \eta}^{z}{ }^{2}\right)+A^{V}\left(\chi_{46}\right) B^{V}\left(\chi_{1}, \chi_{\eta}\right)\left(-4 / 27\left(\chi_{6}-\chi_{4}-3 \chi_{1}\right) \chi_{1} R_{14 \eta}^{z} R_{\eta 61}^{z}\right.$

$\left.-4 / 27\left(2 \chi_{6}+\chi_{4}+3 \chi_{1}\right) \chi_{1} R_{16 \eta}^{z} R_{\eta 61}^{z}\right)+A^{V}\left(\chi_{46}\right) B^{V}\left(\chi_{\eta}\right)\left(-2 / 9\left(3 \chi_{\eta}+\chi_{4}\right) \chi_{1} R_{\eta 61}^{z}{ }^{2}\right)$

$+A^{V}\left(\chi_{\eta}\right)^{2}\left(2 / 9 \chi_{1} R_{\eta 61}^{z}{ }^{4}\right)+A^{V}\left(\chi_{\eta}\right) B^{V}\left(\chi_{1}\right)\left(-2 / 9 \chi_{1} R_{146 \eta}^{z} R_{\eta 61}^{z}+2 / 27 \chi_{1} \chi_{6} R_{14 \eta}^{z}{ }^{2}\right.$

$\left.+1 / 27 \chi_{1} \chi_{4} R_{16 \eta}^{z}{ }^{2}-4 / 9 \chi_{1}^{2} R_{146 \eta}^{c} R_{\eta 61}^{z}{ }^{2}\right)+A^{V}\left(\chi_{\eta}\right) B^{V}\left(\chi_{1}, \chi_{\eta}\right)\left(-8 / 27 \chi_{1} \chi_{6} R_{14 \eta}^{z} R_{\eta 61}^{z}\right.$

$\left.+2 / 27 \chi_{1} \chi_{4} R_{16 \eta}^{z} R_{\eta 61}^{z}+8 / 9 \chi_{1}^{2} R_{\eta 61}^{z}{ }^{4}\right)+A^{V}\left(\chi_{\eta}\right) B^{V}\left(\chi_{\eta}\right)\left(1 / 27\left(8 \chi_{6}+\chi_{4}\right) \chi_{1} R_{\eta 61}^{z}{ }^{2}\right)$

$+A^{V}\left(\chi_{\eta}\right) C^{V}\left(\chi_{1}\right)\left(-4 / 9 \chi_{1}^{2} R_{146 \eta}^{z} R_{\eta 61}^{z}{ }^{2}\right)+B^{V}\left(\chi_{1}\right)^{2}\left(1 / 18 \chi_{1} R_{146 \eta}^{z}{ }^{2}+2 / 9 \chi_{1}^{2} R_{146 \eta}^{z} R_{146 \eta}^{c}\right)$

$+B^{V}\left(\chi_{1}\right) B^{V}\left(\chi_{1}, \chi_{\eta}\right)\left(-4 / 9 \chi_{1}^{2} R_{146 \eta}^{z} R_{\eta 61}^{z}\right)+B^{V}\left(\chi_{1}\right) C^{V}\left(\chi_{1}\right)\left(2 / 9 \chi_{1}^{2} R_{146 \eta}^{z}{ }^{2}\right)$

$+A_{23}^{V}\left(\chi_{1}\right) \frac{1}{16 \pi^{2}}\left(3 / 4 \chi_{1}-1 / 2 \chi_{1} R_{16 \eta}^{z}-1 / 4 \chi_{1} R_{14 \eta}^{z}\right)+A_{23}^{V}\left(\chi_{14}\right) \frac{1}{16 \pi^{2}}\left(1 / 6 \chi_{1}-1 / 3 \chi_{1} R_{\eta 61}^{z}\right.$

$\left.-1 / 3 \chi_{1} R_{\eta 61}^{z}{ }^{2}-1 / 3 \chi_{1} R_{16 \eta}^{z}+1 / 6 \chi_{1} R_{146 \eta}^{c}\right)+A_{23}^{V}\left(\chi_{16}\right) \frac{1}{16 \pi^{2}}\left(1 / 12 \chi_{1}+1 / 3 \chi_{1} R_{\eta 61}^{z}\right.$

$\left.-1 / 6 \chi_{1} R_{\eta 61}^{z}{ }^{2}-1 / 6 \chi_{1} R_{14 \eta}^{z}+1 / 12 \chi_{1} R_{146 \eta}^{c}\right)+H^{V}\left(1, \chi_{1}, \chi_{1}, \chi_{1}, \chi_{1}\right)\left(1 / 3 \chi_{1}^{2}+2 / 9 \chi_{1}^{2} R_{146 \eta}^{c}{ }^{2}\right)$

$+H^{V}\left(1, \chi_{1}, \chi_{1}, \chi_{\eta}, \chi_{1}\right)\left(-8 / 9 \chi_{1}^{2} R_{146 \eta}^{c} R_{\eta 61}^{z}\right)+H^{V}\left(1, \chi_{1}, \chi_{14}, \chi_{14}, \chi_{1}\right)\left(-11 / 18 \chi_{1} R_{146 \eta}^{z}\right.$

$\left.-5 / 6 \chi_{1}^{2} R_{146 \eta}^{c}-1 / 9\left(4 \chi_{4}-7 \chi_{1}\right) \chi_{1} R_{16 \eta}^{z}\right)+H^{V}\left(1, \chi_{1}, \chi_{16}, \chi_{16}, \chi_{1}\right)\left(-11 / 36 \chi_{1} R_{146 \eta}^{z}\right.$

$\left.-5 / 12 \chi_{1}^{2} R_{146 \eta}^{c}-1 / 18\left(4 \chi_{6}-7 \chi_{1}\right) \chi_{1} R_{14 \eta}^{z}\right)+H^{V}\left(1, \chi_{1}, \chi_{\eta}, \chi_{\eta}, \chi_{1}\right)\left(8 / 9 \chi_{1}^{2} R_{\eta 61}^{z}{ }^{4}\right)$

$+H^{V}\left(1, \chi_{14}, \chi_{14}, \chi_{\eta}, \chi_{1}\right)\left(1 / 27\left(\chi_{\eta}-\chi_{4}\right)\left(\chi_{\eta}-\chi_{4}-6 \chi_{1}\right)+4 / 27\left(\chi_{\eta}+2 \chi_{1}\right)\left(\chi_{\eta}-4 \chi_{1}\right) R_{\eta 61}^{z}{ }^{2}\right.$

$\left.+4 / 27\left(\chi_{\eta}^{2}-\chi_{4} \chi_{\eta}-4 \chi_{1} \chi_{\eta}+\chi_{1} \chi_{4}-6 \chi_{1}^{2}\right) R_{\eta 61}^{z}\right)+H^{V}\left(1, \chi_{16}, \chi_{16}, \chi_{\eta}, \chi_{1}\right)\left(2 / 27\left(\chi_{\eta}\right.\right.$

$\left.-\chi_{6}\right)\left(\chi_{\eta}-\chi_{6}-6 \chi_{1}\right)+2 / 27\left(\chi_{\eta}+2 \chi_{1}\right)\left(\chi_{\eta}-4 \chi_{1}\right) R_{\eta 61}^{z}-4 / 27\left(\chi_{\eta}^{2}-\chi_{6} \chi_{\eta}-4 \chi_{1} \chi_{\eta}\right.$

$\left.\left.+\chi_{1} \chi_{6}-6 \chi_{1}^{2}\right) R_{\eta 61}^{z}\right)+H^{V}\left(1, \chi_{4}, \chi_{14}, \chi_{14}, \chi_{1}\right)\left(3 / 4 \chi_{1} \chi_{4}\right)+H^{V}\left(1, \chi_{46}, \chi_{14}, \chi_{16}, \chi_{1}\right)\left(1 / 2\left(\chi_{6}\right.\right.$

$\left.\left.+\chi_{4}\right) \chi_{1}\right)+H^{V}\left(1, \chi_{\eta}, \chi_{14}, \chi_{14}, \chi_{1}\right)\left(1 / 12 \chi_{1} \chi_{\eta}+1 / 3 \chi_{1} \chi_{\eta} R_{\eta 61}^{z}+1 / 3 \chi_{1} \chi_{\eta} R_{\eta 61}^{z}{ }^{2}\right)$

$+H^{V}\left(1, \chi_{\eta}, \chi_{16}, \chi_{16}, \chi_{1}\right)\left(1 / 6 \chi_{1} \chi_{\eta}-1 / 3 \chi_{1} \chi_{\eta} R_{\eta 61}^{z}+1 / 6 \chi_{1} \chi_{\eta} R_{\eta 61}^{z}{ }^{2}\right)$

$+H^{V}\left(2, \chi_{1}, \chi_{1}, \chi_{1}, \chi_{1}\right)\left(4 / 9 \chi_{1}^{2} R_{146 \eta}^{z} R_{146 \eta}^{c}\right)+H^{V}\left(2, \chi_{1}, \chi_{1}, \chi_{\eta}, \chi_{1}\right)\left(-8 / 9 \chi_{1}^{2} R_{146 \eta}^{z} R_{\eta 61}^{z}{ }^{2}\right)$

$+H^{V}\left(2, \chi_{1}, \chi_{14}, \chi_{14}, \chi_{1}\right)\left(-5 / 6 \chi_{1}^{2} R_{146 \eta}^{z}\right)+H^{V}\left(2, \chi_{1}, \chi_{16}, \chi_{16}, \chi_{1}\right)\left(-5 / 12 \chi_{1}^{2} R_{146 \eta}^{z}\right)$

$+H^{V}\left(5, \chi_{1}, \chi_{1}, \chi_{1}, \chi_{1}\right)\left(2 / 9 \chi_{1}^{2} R_{146 \eta}^{z}{ }^{2}\right)+H_{1}^{V}\left(1, \chi_{1}, \chi_{14}, \chi_{14}, \chi_{1}\right)\left(4 / 9 \chi_{1} R_{146 \eta}^{z}+4 / 3 \chi_{1}^{2} R_{146 \eta}^{c}\right.$

$\left.+4 / 9\left(\chi_{4}-4 \chi_{1}\right) \chi_{1} R_{16 \eta}^{z}\right)+H_{1}^{V}\left(1, \chi_{1}, \chi_{16}, \chi_{16}, \chi_{1}\right)\left(2 / 9 \chi_{1} R_{146 \eta}^{z}+2 / 3 \chi_{1}^{2} R_{146 \eta}^{c}\right.$

$\left.+2 / 9\left(\chi_{6}-4 \chi_{1}\right) \chi_{1} R_{14 \eta}^{z}\right)+H_{1}^{V}\left(1, \chi_{14}, \chi_{14}, \chi_{\eta}, \chi_{1}\right)\left(4 / 9\left(\chi_{\eta}-\chi_{4}\right) \chi_{1}+16 / 9\left(\chi_{\eta}\right.\right.$ 
$\left.\left.+2 \chi_{1}\right) \chi_{1} R_{\eta 61}^{z}{ }^{2}+8 / 9\left(2 \chi_{\eta}-\chi_{4}+2 \chi_{1}\right) \chi_{1} R_{\eta 61}^{z}\right)+H_{1}^{V}\left(1, \chi_{16}, \chi_{16}, \chi_{\eta}, \chi_{1}\right)\left(8 / 9\left(\chi_{\eta}-\chi_{6}\right) \chi_{1}\right.$

$\left.+8 / 9\left(\chi_{\eta}+2 \chi_{1}\right) \chi_{1} R_{\eta 61}^{z}-8 / 9\left(2 \chi_{\eta}-\chi_{6}+2 \chi_{1}\right) \chi_{1} R_{\eta 61}^{z}\right)$

$+H_{1}^{V}\left(2, \chi_{1}, \chi_{14}, \chi_{14}, \chi_{1}\right)\left(4 / 3 \chi_{1}^{2} R_{146 \eta}^{z}\right)+H_{1}^{V}\left(2, \chi_{1}, \chi_{16}, \chi_{16}, \chi_{1}\right)\left(2 / 3 \chi_{1}^{2} R_{146 \eta}^{z}\right)$

$+H_{21}^{V}\left(1, \chi_{1}, \chi_{14}, \chi_{14}, \chi_{1}\right)\left(\chi_{1}^{2} R_{16 \eta}^{z}-1 / 2 \chi_{1}^{2} R_{146 \eta}^{c}\right)+H_{21}^{V}\left(1, \chi_{1}, \chi_{16}, \chi_{16}, \chi_{1}\right)\left(1 / 2 \chi_{1}^{2} R_{14 \eta}^{z}\right.$

$\left.-1 / 4 \chi_{1}^{2} R_{146 \eta}^{c}\right)+H_{21}^{V}\left(1, \chi_{4}, \chi_{14}, \chi_{14}, \chi_{1}\right)\left(9 / 4 \chi_{1}^{2}\right)+H_{21}^{V}\left(1, \chi_{46}, \chi_{14}, \chi_{16}, \chi_{1}\right)\left(3 \chi_{1}^{2}\right)$

$+H_{21}^{V}\left(1, \chi_{\eta}, \chi_{14}, \chi_{14}, \chi_{1}\right)\left(1 / 4 \chi_{1}^{2}+\chi_{1}^{2} R_{\eta 61}^{z}+\chi_{1}^{2} R_{\eta 61}^{z}{ }^{2}\right)+H_{21}^{V}\left(1, \chi_{\eta}, \chi_{16}, \chi_{16}, \chi_{1}\right)\left(1 / 2 \chi_{1}^{2}\right.$

$\left.-\chi_{1}^{2} R_{\eta 61}^{z}+1 / 2 \chi_{1}^{2} R_{\eta 61}^{z}{ }^{2}\right)+H_{21}^{V}\left(2, \chi_{1}, \chi_{14}, \chi_{14}, \chi_{1}\right)\left(-1 / 2 \chi_{1}^{2} R_{146 \eta}^{z}\right)$

$+H_{21}^{V}\left(2, \chi_{1}, \chi_{16}, \chi_{16}, \chi_{1}\right)\left(-1 / 4 \chi_{1}^{2} R_{146 \eta}^{z}\right)+H_{27}^{V}\left(1, \chi_{1}, \chi_{14}, \chi_{14}, \chi_{1}\right)\left(-\chi_{1} R_{16 \eta}^{z}\right.$

$\left.+1 / 2 \chi_{1} R_{146 \eta}^{c}\right)+H_{27}^{V}\left(1, \chi_{1}, \chi_{16}, \chi_{16}, \chi_{1}\right)\left(-1 / 2 \chi_{1} R_{14 \eta}^{z}+1 / 4 \chi_{1} R_{146 \eta}^{c}\right)$

$+H_{27}^{V}\left(1, \chi_{4}, \chi_{14}, \chi_{14}, \chi_{1}\right)\left(-9 / 4 \chi_{1}\right)+H_{27}^{V}\left(1, \chi_{46}, \chi_{14}, \chi_{16}, \chi_{1}\right)\left(-3 \chi_{1}\right)$

$+H_{27}^{V}\left(1, \chi_{\eta}, \chi_{14}, \chi_{14}, \chi_{1}\right)\left(-1 / 4 \chi_{1}-\chi_{1} R_{\eta 61}^{z}-\chi_{1} R_{\eta 61}^{z}{ }^{2}\right)$

$+H_{27}^{V}\left(1, \chi_{\eta}, \chi_{16}, \chi_{16}, \chi_{1}\right)\left(-1 / 2 \chi_{1}+\chi_{1} R_{\eta 61}^{z}-1 / 2 \chi_{1} R_{\eta 61}^{z}{ }^{2}\right)$

$+H_{27}^{V}\left(2, \chi_{1}, \chi_{14}, \chi_{14}, \chi_{1}\right)\left(1 / 2 \chi_{1} R_{146 \eta}^{z}\right)+H_{27}^{V}\left(2, \chi_{1}, \chi_{16}, \chi_{16}, \chi_{1}\right)\left(1 / 4 \chi_{1} R_{146 \eta}^{z}\right)$

\section{B Expressions for the decay constant}

$$
F_{0} \Delta^{V} F_{12}^{2(4) 12}=+A^{V}\left(\chi_{14}\right)(1)+A^{V}\left(\chi_{16}\right)(1 / 2)
$$

$F_{0}^{3} \Delta^{V} F_{12}^{2(6 L) 12}=+A^{V}\left(\chi_{1}\right)\left(-4 / 3 \hat{L}_{5}^{r} \chi_{1} R_{146 \eta}^{c}+4 \hat{L}_{3}^{r} R_{146 \eta}^{z}+4 \hat{L}_{3}^{r} \chi_{1} R_{146 \eta}^{c}-10 \hat{L}_{2}^{r} \chi_{1}-4 \hat{L}_{1}^{r} \chi_{1}\right.$

$\left.+4 \hat{L}_{0}^{r} R_{146 \eta}^{z}+4 \hat{L}_{0}^{r} \chi_{1} R_{146 \eta}^{c}\right)+A^{V}\left(\chi_{14}\right)\left(4 \hat{L}_{5}^{r} \chi_{1}-4\left(\chi_{6}+2 \chi_{4}\right) \hat{L}_{4}^{r}-10\left(\chi_{4}+\chi_{1}\right) \hat{L}_{3}^{r}\right.$

$\left.-4\left(\chi_{4}+\chi_{1}\right) \hat{L}_{0}^{r}\right)+A^{V}\left(\chi_{16}\right)\left(2 \hat{L}_{5}^{r} \chi_{1}-2\left(\chi_{6}+2 \chi_{4}\right) \hat{L}_{4}^{r}-5\left(\chi_{6}+\chi_{1}\right) \hat{L}_{3}^{r}-2\left(\chi_{6}+\chi_{1}\right) \hat{L}_{0}^{r}\right)$

$+A^{V}\left(\chi_{4}\right)\left(12 \hat{L}_{4}^{r} \chi_{4}-6 \hat{L}_{2}^{r} \chi_{4}-24 \hat{L}_{1}^{r} \chi_{4}\right)+A^{V}\left(\chi_{46}\right)\left(8\left(\chi_{6}+\chi_{4}\right) \hat{L}_{4}^{r}-4\left(\chi_{6}+\chi_{4}\right) \hat{L}_{2}^{r}\right.$

$\left.-16\left(\chi_{6}+\chi_{4}\right) \hat{L}_{1}^{r}\right)+A^{V}\left(\chi_{\eta}\right)\left(8 / 3 \hat{L}_{5}^{r} \chi_{1} R_{\eta 61}^{z}{ }^{2}-8 \hat{L}_{3}^{r} \chi_{\eta} R_{\eta 61}^{z}{ }^{2}-2 \hat{L}_{2}^{r} \chi_{\eta}-8 \hat{L}_{1}^{r} \chi_{\eta}\right.$

$\left.-8 \hat{L}_{0}^{r} \chi_{\eta} R_{\eta 61}^{z}{ }^{2}+4 / 3\left(2 \chi_{6}+\chi_{4}\right) \hat{L}_{4}^{r}\right)+B^{V}\left(\chi_{1}\right)\left(-4 / 3 \hat{L}_{5}^{r} \chi_{1} R_{146 \eta}^{z}+4 \hat{L}_{3}^{r} \chi_{1} R_{146 \eta}^{z}\right.$

$\left.+4 \hat{L}_{0}^{r} \chi_{1} R_{146 \eta}^{z}\right)+B^{V}\left(\chi_{14}\right)\left(8\left(\chi_{4}+\chi_{1}\right)\left(\chi_{6}+2 \chi_{4}\right) \hat{L}_{6}^{r}-4\left(\chi_{4}+\chi_{1}\right)\left(\chi_{6}+2 \chi_{4}\right) \hat{L}_{4}^{r}\right.$

$\left.+4\left(\chi_{4}+\chi_{1}\right)^{2} \hat{L}_{8}^{r}-2\left(\chi_{4}+\chi_{1}\right)^{2} \hat{L}_{5}^{r}\right)+B^{V}\left(\chi_{16}\right)\left(4\left(\chi_{6}+\chi_{1}\right)\left(\chi_{6}+2 \chi_{4}\right) \hat{L}_{6}^{r}\right.$

$\left.-2\left(\chi_{6}+\chi_{1}\right)\left(\chi_{6}+2 \chi_{4}\right) \hat{L}_{4}^{r}+2\left(\chi_{6}+\chi_{1}\right)^{2} \hat{L}_{8}^{r}-\left(\chi_{6}+\chi_{1}\right)^{2} \hat{L}_{5}^{r}\right)+A_{23}^{V}\left(\chi_{1}\right)\left(-4 \hat{L}_{3}^{r} R_{146 \eta}^{c}\right.$

$\left.+6 \hat{L}_{2}^{r}+12 \hat{L}_{1}^{r}-4 \hat{L}_{0}^{r} R_{146 \eta}^{c}\right)+A_{23}^{V}\left(\chi_{14}\right)\left(12 \hat{L}_{3}^{r}+24 \hat{L}_{0}^{r}\right)+A_{23}^{V}\left(\chi_{16}\right)\left(6 \hat{L}_{3}^{r}+12 \hat{L}_{0}^{r}\right)$

$+A_{23}^{V}\left(\chi_{4}\right)\left(18 \hat{L}_{2}^{r}\right)+A_{23}^{V}\left(\chi_{46}\right)\left(24 \hat{L}_{2}^{r}\right)+A_{23}^{V}\left(\chi_{\eta}\right)\left(8 \hat{L}_{3}^{r} R_{\eta 61}^{z}+6 \hat{L}_{2}^{r}+8 \hat{L}_{0}^{r} R_{\eta 61}^{z}{ }^{2}\right)$ 


$$
+B_{23}^{V}\left(\chi_{1}\right)\left(-4 \hat{L}_{3}^{r} R_{146 \eta}^{z}-4 \hat{L}_{0}^{r} R_{146 \eta}^{z}\right)
$$

$$
\begin{aligned}
& F_{0}^{3} \Delta^{V} F_{12}^{2(6 R) 12}=+\bar{A}\left(\chi_{1}\right) B^{V}\left(\chi_{14}\right)\left(1 / 18 R_{146 \eta}^{z}-1 / 9\left(\chi_{4}+2 \chi_{1}\right) R_{16 \eta}^{z}\right) \\
& +\bar{A}\left(\chi_{1}\right) B^{V}\left(\chi_{16}\right)\left(1 / 36 R_{146 \eta}^{z}-1 / 18\left(\chi_{6}+2 \chi_{1}\right) R_{14 \eta}^{z}\right) \\
& +\bar{A}\left(\chi_{14}\right) A^{V}\left(\chi_{14}\right)\left(5 / 54-5 / 27 R_{\eta 61}^{z}-5 / 27 R_{\eta 61}^{z}{ }^{2}-5 / 27 R_{16 \eta}^{z}+5 / 54 R_{146 \eta}^{c}\right) \\
& +\bar{A}\left(\chi_{16}\right) A^{V}\left(\chi_{16}\right)\left(5 / 108+5 / 27 R_{\eta 61}^{z}-5 / 54 R_{\eta 61}^{z}{ }^{2}-5 / 54 R_{14 \eta}^{z}+5 / 108 R_{146 \eta}^{c}\right) \\
& +\bar{A}\left(\chi_{\eta}\right) B^{V}\left(\chi_{14}\right)\left(-1 / 36\left(\chi_{\eta}-\chi_{4}\right)-1 / 9\left(\chi_{\eta}+\chi_{4}+\chi_{1}\right) R_{\eta 61}^{z}-1 / 9\left(\chi_{\eta}-\chi_{1}\right) R_{\eta 61}^{z}{ }^{2}\right) \\
& +\bar{A}\left(\chi_{\eta}\right) B^{V}\left(\chi_{16}\right)\left(-1 / 18\left(\chi_{\eta}-\chi_{6}\right)+1 / 9\left(\chi_{\eta}+\chi_{6}+\chi_{1}\right) R_{\eta 61}^{z}-1 / 18\left(\chi_{\eta}-\chi_{1}\right) R_{\eta 61}^{z}{ }^{2}\right) \\
& +\bar{B}\left(\chi_{14}\right) A^{V}\left(\chi_{1}\right)\left(1 / 18 R_{146 \eta}^{z}-1 / 9\left(\chi_{4}+2 \chi_{1}\right) R_{16 \eta}^{z}\right)+\bar{B}\left(\chi_{14}\right) A^{V}\left(\chi_{\eta}\right)\left(-1 / 36\left(\chi_{\eta}-\chi_{4}\right)\right. \\
& \left.-1 / 9\left(\chi_{\eta}+\chi_{4}+\chi_{1}\right) R_{\eta 61}^{z}-1 / 9\left(\chi_{\eta}-\chi_{1}\right) R_{\eta 61}^{z}{ }^{2}\right)+\bar{B}\left(\chi_{16}\right) A^{V}\left(\chi_{1}\right)\left(1 / 36 R_{146 \eta}^{z}\right. \\
& \left.-1 / 18\left(\chi_{6}+2 \chi_{1}\right) R_{14 \eta}^{z}\right)+\bar{B}\left(\chi_{16}\right) A^{V}\left(\chi_{\eta}\right)\left(-1 / 18\left(\chi_{\eta}-\chi_{6}\right)+1 / 9\left(\chi_{\eta}+\chi_{6}+\chi_{1}\right) R_{\eta 61}^{z}\right. \\
& \left.-1 / 18\left(\chi_{\eta}-\chi_{1}\right) R_{\eta 61}^{z}{ }^{2}\right)+A^{V}\left(\chi_{1}\right) \frac{1}{16 \pi^{2}}\left(1 / 8 \chi_{1}-1 / 12 \chi_{1} R_{16 \eta}^{z}-1 / 24 \chi_{1} R_{14 \eta}^{z}\right) \\
& +A^{V}\left(\chi_{1}\right) B^{V}\left(\chi_{14}\right)\left(1 / 18 R_{146 \eta}^{z}-1 / 9\left(\chi_{4}+2 \chi_{1}\right) R_{16 \eta}^{z}\right)+A^{V}\left(\chi_{1}\right) B^{V}\left(\chi_{16}\right)\left(1 / 36 R_{146 \eta}^{z}\right. \\
& \left.-1 / 18\left(\chi_{6}+2 \chi_{1}\right) R_{14 \eta}^{z}\right)+A^{V}\left(\chi_{14}\right) \frac{1}{16 \pi^{2}}\left(1 / 12 R_{146 \eta}^{z}-1 / 24\left(\chi_{\eta}+6 \chi_{6}+14 \chi_{4}+5 \chi_{1}\right)\right. \\
& -1 / 12\left(2 \chi_{\eta}+\chi_{4}+\chi_{1}\right) R_{\eta 61}^{z}-1 / 12\left(2 \chi_{\eta}+\chi_{4}+\chi_{1}\right) R_{\eta 61}^{z}{ }^{2}-1 / 12\left(\chi_{4}+3 \chi_{1}\right) R_{16 \eta}^{z} \\
& \left.+1 / 24\left(\chi_{4}+3 \chi_{1}\right) R_{146 \eta}^{c}\right)+A^{V}\left(\chi_{14}\right)^{2}\left(5 / 108-5 / 54 R_{\eta 61}^{z}-5 / 54 R_{\eta 61}^{z}{ }^{2}-5 / 54 R_{16 \eta}^{z}\right. \\
& \left.+5 / 108 R_{146 \eta}^{c}\right)+A^{V}\left(\chi_{16}\right) \frac{1}{16 \pi^{2}}\left(1 / 24 R_{146 \eta}^{z}+1 / 12\left(2 \chi_{\eta}+\chi_{6}+\chi_{1}\right) R_{\eta 61}^{z}\right. \\
& -1 / 24\left(2 \chi_{\eta}+\chi_{6}+\chi_{1}\right) R_{\eta 61}^{z}{ }^{2}-1 / 48\left(4 \chi_{\eta}+5 \chi_{6}+12 \chi_{4}+5 \chi_{1}\right)-1 / 24\left(\chi_{6}+3 \chi_{1}\right) R_{14 \eta}^{z} \\
& \left.+1 / 48\left(\chi_{6}+3 \chi_{1}\right) R_{146 \eta}^{c}\right)+A^{V}\left(\chi_{16}\right)^{2}\left(5 / 216+5 / 54 R_{\eta 61}^{z}-5 / 108 R_{\eta 61}^{z}{ }^{2}-5 / 108 R_{14 \eta}^{z}\right. \\
& \left.+5 / 216 R_{146 \eta}^{c}\right)+A^{V}\left(\chi_{\eta}\right) B^{V}\left(\chi_{14}\right)\left(-1 / 36\left(\chi_{\eta}-\chi_{4}\right)-1 / 9\left(\chi_{\eta}+\chi_{4}+\chi_{1}\right) R_{\eta 61}^{z}\right. \\
& \left.-1 / 9\left(\chi_{\eta}-\chi_{1}\right) R_{\eta 61}^{z}{ }^{2}\right)+A^{V}\left(\chi_{\eta}\right) B^{V}\left(\chi_{16}\right)\left(-1 / 18\left(\chi_{\eta}-\chi_{6}\right)+1 / 9\left(\chi_{\eta}+\chi_{6}+\chi_{1}\right) R_{\eta 61}^{z}\right. \\
& \left.-1 / 18\left(\chi_{\eta}-\chi_{1}\right) R_{\eta 61}^{z}{ }^{2}\right)+A_{23}^{V}\left(\chi_{1}\right) \frac{1}{16 \pi^{2}}\left(-3 / 8+1 / 4 R_{16 \eta}^{z}+1 / 8 R_{14 \eta}^{z}\right) \\
& +A_{23}^{V}\left(\chi_{14}\right) \frac{1}{16 \pi^{2}}\left(-1 / 12+1 / 6 R_{\eta 61}^{z}+1 / 6 R_{\eta 61}^{z}{ }^{2}+1 / 6 R_{16 \eta}^{z}-1 / 12 R_{146 \eta}^{c}\right) \\
& +A_{23}^{V}\left(\chi_{16}\right) \frac{1}{16 \pi^{2}}\left(-1 / 24-1 / 6 R_{\eta 61}^{z}+1 / 12 R_{\eta 61}^{z}{ }^{2}+1 / 12 R_{14 \eta}^{z}-1 / 24 R_{146 \eta}^{c}\right) \\
& +H^{V}\left(1, \chi_{1}, \chi_{14}, \chi_{14}, \chi_{1}\right)\left(1 / 12 R_{146 \eta}^{z}-1 / 6 \chi_{1} R_{16 \eta}^{z}+1 / 12 \chi_{1} R_{146 \eta}^{c}\right) \\
& +H^{V}\left(1, \chi_{1}, \chi_{16}, \chi_{16}, \chi_{1}\right)\left(1 / 24 R_{146 \eta}^{z}-1 / 12 \chi_{1} R_{14 \eta}^{z}+1 / 24 \chi_{1} R_{146 \eta}^{c}\right) \\
& +H^{V}\left(1, \chi_{4}, \chi_{14}, \chi_{14}, \chi_{1}\right)\left(-3 / 8 \chi_{4}\right)+H^{V}\left(1, \chi_{46}, \chi_{14}, \chi_{16}, \chi_{1}\right)\left(-1 / 4\left(\chi_{6}+\chi_{4}\right)\right)
\end{aligned}
$$


$+H^{V}\left(1, \chi_{\eta}, \chi_{14}, \chi_{14}, \chi_{1}\right)\left(-1 / 24 \chi_{\eta}-1 / 6 \chi_{\eta} R_{\eta 61}^{z}-1 / 6 \chi_{\eta} R_{\eta 61}^{z}{ }^{2}\right)$

$+H^{V}\left(1, \chi_{\eta}, \chi_{16}, \chi_{16}, \chi_{1}\right)\left(-1 / 12 \chi_{\eta}+1 / 6 \chi_{\eta} R_{\eta 61}^{z}-1 / 12 \chi_{\eta} R_{\eta 61}^{z}{ }^{2}\right)$

$+H^{V}\left(2, \chi_{1}, \chi_{14}, \chi_{14}, \chi_{1}\right)\left(5 / 54 \chi_{1} R_{146 \eta}^{z}\right)+H^{V}\left(2, \chi_{1}, \chi_{16}, \chi_{16}, \chi_{1}\right)\left(5 / 108 \chi_{1} R_{146 \eta}^{z}\right)$

$+H_{1}^{V}\left(2, \chi_{1}, \chi_{14}, \chi_{14}, \chi_{1}\right)\left(-1 / 108 \chi_{1} R_{146 \eta}^{z}\right)+H_{1}^{V}\left(2, \chi_{1}, \chi_{16}, \chi_{16}, \chi_{1}\right)\left(-1 / 216 \chi_{1} R_{146 \eta}^{z}\right)$

$+H_{1}^{V}\left(3, \chi_{14}, \chi_{1}, \chi_{14}, \chi_{1}\right)\left(-1 / 54 \chi_{1} R_{146 \eta}^{z}\right)+H_{1}^{V}\left(3, \chi_{16}, \chi_{1}, \chi_{16}, \chi_{1}\right)\left(-1 / 108 \chi_{1} R_{146 \eta}^{z}\right)$

$+H_{27}^{V}\left(1, \chi_{1}, \chi_{14}, \chi_{14}, \chi_{1}\right)\left(1 / 2 R_{16 \eta}^{z}-1 / 4 R_{146 \eta}^{c}\right)+H_{27}^{V}\left(1, \chi_{1}, \chi_{16}, \chi_{16}, \chi_{1}\right)\left(1 / 4 R_{14 \eta}^{z}-1 / 8 R_{146 \eta}^{c}\right)$

$+H_{27}^{V}\left(1, \chi_{4}, \chi_{14}, \chi_{14}, \chi_{1}\right)(9 / 8)+H_{27}^{V}\left(1, \chi_{46}, \chi_{14}, \chi_{16}, \chi_{1}\right)(3 / 2)$

$+H_{27}^{V}\left(1, \chi_{\eta}, \chi_{14}, \chi_{14}, \chi_{1}\right)\left(1 / 8+1 / 2 R_{\eta 61}^{z}+1 / 2 R_{\eta 61}^{z}{ }^{2}\right)$

$+H_{27}^{V}\left(1, \chi_{\eta}, \chi_{16}, \chi_{16}, \chi_{1}\right)\left(1 / 4-1 / 2 R_{\eta 61}^{z}+1 / 4 R_{\eta 61}^{z}{ }^{2}\right)$

$+H_{27}^{V}\left(2, \chi_{1}, \chi_{14}, \chi_{14}, \chi_{1}\right)\left(-1 / 4 R_{146 \eta}^{z}\right)+H_{27}^{V}\left(2, \chi_{1}, \chi_{16}, \chi_{16}, \chi_{1}\right)\left(-1 / 8 R_{146 \eta}^{z}\right)$

$+H^{\prime} V\left(1, \chi_{1}, \chi_{1}, \chi_{1}, \chi_{1}\right)\left(1 / 6 \chi_{1}^{2}+1 / 9 \chi_{1}^{2} R_{146 \eta}^{c}{ }^{2}\right)+H^{\prime} V\left(1, \chi_{1}, \chi_{1}, \chi_{\eta}, \chi_{1}\right)\left(-4 / 9 \chi_{1}^{2} R_{146 \eta}^{c} R_{\eta 61}^{z}{ }^{2}\right)$

$+H^{\prime} V\left(1, \chi_{1}, \chi_{14}, \chi_{14}, \chi_{1}\right)\left(-11 / 36 \chi_{1} R_{146 \eta}^{z}-5 / 12 \chi_{1}^{2} R_{146 \eta}^{c}-1 / 18\left(4 \chi_{4}-7 \chi_{1}\right) \chi_{1} R_{16 \eta}^{z}\right)$

$+H^{\prime} V\left(1, \chi_{1}, \chi_{16}, \chi_{16}, \chi_{1}\right)\left(-11 / 72 \chi_{1} R_{146 \eta}^{z}-5 / 24 \chi_{1}^{2} R_{146 \eta}^{c}-1 / 36\left(4 \chi_{6}-7 \chi_{1}\right) \chi_{1} R_{14 \eta}^{z}\right)$

$+H^{\prime} V\left(1, \chi_{1}, \chi_{\eta}, \chi_{\eta}, \chi_{1}\right)\left(4 / 9 \chi_{1}^{2} R_{\eta 61}^{z}{ }^{4}\right)+H^{\prime} V\left(1, \chi_{14}, \chi_{14}, \chi_{\eta}, \chi_{1}\right)\left(1 / 54\left(\chi_{\eta}-\chi_{4}\right)\left(\chi_{\eta}-\chi_{4}\right.\right.$

$\left.\left.-6 \chi_{1}\right)+2 / 27\left(\chi_{\eta}+2 \chi_{1}\right)\left(\chi_{\eta}-4 \chi_{1}\right) R_{\eta 61}^{z}{ }^{2}+2 / 27\left(\chi_{\eta}^{2}-\chi_{4} \chi_{\eta}-4 \chi_{1} \chi_{\eta}+\chi_{1} \chi_{4}-6 \chi_{1}^{2}\right) R_{\eta 61}^{z}\right)$

$+H^{\prime} V\left(1, \chi_{16}, \chi_{16}, \chi_{\eta}, \chi_{1}\right)\left(1 / 27\left(\chi_{\eta}-\chi_{6}\right)\left(\chi_{\eta}-\chi_{6}-6 \chi_{1}\right)+1 / 27\left(\chi_{\eta}+2 \chi_{1}\right)\left(\chi_{\eta}-4 \chi_{1}\right)\right.$

$\left.R_{\eta 61}^{z}{ }^{2}-2 / 27\left(\chi_{\eta}^{2}-\chi_{6} \chi_{\eta}-4 \chi_{1} \chi_{\eta}+\chi_{1} \chi_{6}-6 \chi_{1}^{2}\right) R_{\eta 61}^{z}\right)+H^{\prime} V\left(1, \chi_{4}, \chi_{14}, \chi_{14}, \chi_{1}\right)\left(3 / 8 \chi_{1} \chi_{4}\right)$

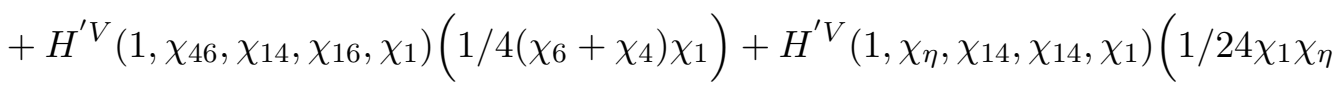

$\left.+1 / 6 \chi_{1} \chi_{\eta} R_{\eta 61}^{z}+1 / 6 \chi_{1} \chi_{\eta} R_{\eta 61}^{z}{ }^{2}\right)+H^{\prime}{ }^{\prime}\left(1, \chi_{\eta}, \chi_{16}, \chi_{16}, \chi_{1}\right)\left(1 / 12 \chi_{1} \chi_{\eta}-1 / 6 \chi_{1} \chi_{\eta} R_{\eta 61}^{z}\right.$

$\left.+1 / 12 \chi_{1} \chi_{\eta} R_{\eta 61}^{z}{ }^{2}\right)+H^{\prime} V\left(2, \chi_{1}, \chi_{1}, \chi_{1}, \chi_{1}\right)\left(2 / 9 \chi_{1}^{2} R_{146 \eta}^{z} R_{146 \eta}^{c}\right)$

$+H^{\prime} V\left(2, \chi_{1}, \chi_{1}, \chi_{\eta}, \chi_{1}\right)\left(-4 / 9 \chi_{1}^{2} R_{146 \eta}^{z} R_{\eta 61}^{z}\right)+H^{\prime} V\left(2, \chi_{1}, \chi_{14}, \chi_{14}, \chi_{1}\right)\left(-5 / 12 \chi_{1}^{2} R_{146 \eta}^{z}\right)$

$+H^{\prime} V\left(2, \chi_{1}, \chi_{16}, \chi_{16}, \chi_{1}\right)\left(-5 / 24 \chi_{1}^{2} R_{146 \eta}^{z}\right)+H^{\prime} V\left(5, \chi_{1}, \chi_{1}, \chi_{1}, \chi_{1}\right)\left(1 / 9 \chi_{1}^{2} R_{146 \eta}^{z}{ }^{2}\right)$

$+H_{1}^{\prime} V\left(1, \chi_{1}, \chi_{14}, \chi_{14}, \chi_{1}\right)\left(2 / 9 \chi_{1} R_{146 \eta}^{z}+2 / 3 \chi_{1}^{2} R_{146 \eta}^{c}+2 / 9\left(\chi_{4}-4 \chi_{1}\right) \chi_{1} R_{16 \eta}^{z}\right)$

$+H_{1}^{\prime} V\left(1, \chi_{1}, \chi_{16}, \chi_{16}, \chi_{1}\right)\left(1 / 9 \chi_{1} R_{146 \eta}^{z}+1 / 3 \chi_{1}^{2} R_{146 \eta}^{c}+1 / 9\left(\chi_{6}-4 \chi_{1}\right) \chi_{1} R_{14 \eta}^{z}\right)$

$+H_{1}^{\prime} V\left(1, \chi_{14}, \chi_{14}, \chi_{\eta}, \chi_{1}\right)\left(2 / 9\left(\chi_{\eta}-\chi_{4}\right) \chi_{1}+8 / 9\left(\chi_{\eta}+2 \chi_{1}\right) \chi_{1} R_{\eta 61}^{z}{ }^{2}+4 / 9\left(2 \chi_{\eta}-\chi_{4}\right.\right.$

$\left.\left.+2 \chi_{1}\right) \chi_{1} R_{\eta 61}^{z}\right)+H_{1}^{\prime} V\left(1, \chi_{16}, \chi_{16}, \chi_{\eta}, \chi_{1}\right)\left(4 / 9\left(\chi_{\eta}-\chi_{6}\right) \chi_{1}+4 / 9\left(\chi_{\eta}+2 \chi_{1}\right) \chi_{1} R_{\eta 61}^{z}{ }^{2}\right.$

$\left.-4 / 9\left(2 \chi_{\eta}-\chi_{6}+2 \chi_{1}\right) \chi_{1} R_{\eta 61}^{z}\right)+H_{1}^{\prime V}\left(2, \chi_{1}, \chi_{14}, \chi_{14}, \chi_{1}\right)\left(2 / 3 \chi_{1}^{2} R_{146 \eta}^{z}\right)$ 


$$
\begin{aligned}
& +H_{1}^{\prime V}\left(2, \chi_{1}, \chi_{16}, \chi_{16}, \chi_{1}\right)\left(1 / 3 \chi_{1}^{2} R_{146 \eta}^{z}\right)+H_{21}^{\prime}\left(1, \chi_{1}, \chi_{14}, \chi_{14}, \chi_{1}\right)\left(1 / 2 \chi_{1}^{2} R_{16 \eta}^{z}-1 / 4 \chi_{1}^{2} R_{146 \eta}^{c}\right) \\
& +H_{21}^{\prime V}\left(1, \chi_{1}, \chi_{16}, \chi_{16}, \chi_{1}\right)\left(1 / 4 \chi_{1}^{2} R_{14 \eta}^{z}-1 / 8 \chi_{1}^{2} R_{146 \eta}^{c}\right)+H_{21}^{\prime}\left(1, \chi_{4}, \chi_{14}, \chi_{14}, \chi_{1}\right)\left(9 / 8 \chi_{1}^{2}\right) \\
& +H_{21}^{\prime V}\left(1, \chi_{46}, \chi_{14}, \chi_{16}, \chi_{1}\right)\left(3 / 2 \chi_{1}^{2}\right)+H_{21}^{\prime}\left(1, \chi_{\eta}, \chi_{14}, \chi_{14}, \chi_{1}\right)\left(1 / 8 \chi_{1}^{2}+1 / 2 \chi_{1}^{2} R_{\eta 61}^{z}\right. \\
& \left.+1 / 2 \chi_{1}^{2} R_{\eta 61}^{z}\right)+H_{21}^{\prime} V\left(1, \chi_{\eta}, \chi_{16}, \chi_{16}, \chi_{1}\right)\left(1 / 4 \chi_{1}^{2}-1 / 2 \chi_{1}^{2} R_{\eta 61}^{z}+1 / 4 \chi_{1}^{2} R_{\eta 61}^{z}{ }^{2}\right) \\
& +H_{21}^{\prime V}\left(2, \chi_{1}, \chi_{14}, \chi_{14}, \chi_{1}\right)\left(-1 / 4 \chi_{1}^{2} R_{146 \eta}^{z}\right)+H_{21}^{\prime}\left(2, \chi_{1}, \chi_{16}, \chi_{16}, \chi_{1}\right)\left(-1 / 8 \chi_{1}^{2} R_{146 \eta}^{z}\right) \\
& +H_{27}^{\prime}\left(1, \chi_{1}, \chi_{14}, \chi_{14}, \chi_{1}\right)\left(-1 / 2 \chi_{1} R_{16 \eta}^{z}+1 / 4 \chi_{1} R_{146 \eta}^{c}\right) \\
& +H_{27}^{\prime}\left(1, \chi_{1}, \chi_{16}, \chi_{16}, \chi_{1}\right)\left(-1 / 4 \chi_{1} R_{14 \eta}^{z}+1 / 8 \chi_{1} R_{146 \eta}^{c}\right)+H_{27}^{\prime} V\left(1, \chi_{4}, \chi_{14}, \chi_{14}, \chi_{1}\right)\left(-9 / 8 \chi_{1}\right) \\
& +H_{27}^{\prime} V\left(1, \chi_{46}, \chi_{14}, \chi_{16}, \chi_{1}\right)\left(-3 / 2 \chi_{1}\right)+H_{27}^{\prime}{ }^{\prime}\left(1, \chi_{\eta}, \chi_{14}, \chi_{14}, \chi_{1}\right)\left(-1 / 8 \chi_{1}-1 / 2 \chi_{1} R_{\eta 61}^{z}\right. \\
& \left.-1 / 2 \chi_{1} R_{\eta 61}^{z}{ }^{2}\right)+H_{27}^{\prime}\left(1, \chi_{\eta}, \chi_{16}, \chi_{16}, \chi_{1}\right)\left(-1 / 4 \chi_{1}+1 / 2 \chi_{1} R_{\eta 61}^{z}-1 / 4 \chi_{1} R_{\eta 61}^{z}\right) \\
& +H_{27}^{\prime} V\left(2, \chi_{1}, \chi_{14}, \chi_{14}, \chi_{1}\right)\left(1 / 4 \chi_{1} R_{146 \eta}^{z}\right)+H_{27}^{\prime} V\left(2, \chi_{1}, \chi_{16}, \chi_{16}, \chi_{1}\right)\left(1 / 8 \chi_{1} R_{146 \eta}^{z}\right)
\end{aligned}
$$

Open Access. This article is distributed under the terms of the Creative Commons Attribution License (CC-BY 4.0), which permits any use, distribution and reproduction in any medium, provided the original author(s) and source are credited.

\section{References}

[1] S. Aoki et al., Review of lattice results concerning low-energy particle physics, Eur. Phys. J. C 74 (2014) 2890 [arXiv:1310.8555] [INSPIRE].

[2] S. Weinberg, Phenomenological Lagrangians, Physica A 96 (1979) 327 [InSPIRE].

[3] J. Gasser and H. Leutwyler, Chiral Perturbation Theory to One Loop, Annals Phys. 158 (1984) 142 [INSPIRE].

[4] J. Gasser and H. Leutwyler, Chiral Perturbation Theory: Expansions in the Mass of the Strange Quark, Nucl. Phys. B 250 (1985) 465 [InSPIRE].

[5] J. Gasser and H. Leutwyler, Light Quarks at Low Temperatures, Phys. Lett. B 184 (1987) 83 [INSPIRE].

[6] J. Gasser and H. Leutwyler, Thermodynamics of Chiral Symmetry, Phys. Lett. B 188 (1987) 477 [INSPIRE].

[7] J. Gasser and H. Leutwyler, Spontaneously Broken Symmetries: Effective Lagrangians at Finite Volume, Nucl. Phys. B 307 (1988) 763 [INSPIRE].

[8] C.W. Bernard and M.F.L. Golterman, Partially quenched gauge theories and an application to staggered fermions, Phys. Rev. D 49 (1994) 486 [hep-lat/9306005] [INSPIRE].

[9] C. Bernard and M. Golterman, On the foundations of partially quenched chiral perturbation theory, Phys. Rev. D 88 (2013) 014004 [arXiv:1304.1948] [INSPIRE].

[10] S.R. Sharpe and N. Shoresh, Physical results from unphysical simulations, Phys. Rev. D 62 (2000) 094503 [hep-lat/0006017] [INSPIRE]. 
[11] J. Bijnens, N. Danielsson and T.A. Lähde, The pseudoscalar meson mass to two loops in three-flavor partially quenched chiPT, Phys. Rev. D 70 (2004) 111503 [hep-lat/0406017] [INSPIRE].

[12] J. Bijnens and T.A. Lähde, Decay constants of pseudoscalar mesons to two loops in three-flavor partially quenched (chi)PT, Phys. Rev. D 71 (2005) 094502 [hep-lat/0501014] [INSPIRE].

[13] J. Bijnens, N. Danielsson and T.A. Lähde, Three-flavor partially quenched chiral perturbation theory at NNLO for meson masses and decay constants, Phys. Rev. D 73 (2006) 074509 [hep-lat/0602003] [INSPIRE].

[14] J. Bijnens and T.A. Lähde, Masses and decay constants of pseudoscalar mesons to two loops in two-flavor partially quenched chiral perturbation theory, Phys. Rev. D 72 (2005) 074502 [hep-lat/0506004] [INSPIRE].

[15] J. Bijnens and T. Rössler, Finite Volume at Two-loops in Chiral Perturbation Theory, JHEP 01 (2015) 034 [arXiv:1411.6384] [INSPIRE].

[16] J. Bijnens, E. Boström and T.A. Lähde, Two-loop Sunset Integrals at Finite Volume, JHEP 01 (2014) 019 [arXiv:1311.3531] [InSPIRE].

[17] G. Colangelo and C. Haefeli, Finite volume effects for the pion mass at two loops, Nucl. Phys. B 744 (2006) 14 [hep-lat/0602017] [INSPIRE].

[18] J. Bijnens and K. Ghorbani, Finite volume dependence of the quark-antiquark vacuum expectation value, Phys. Lett. B 636 (2006) 51 [hep-lat/0602019] [INSPIRE].

[19] P.H. Damgaard and H. Fukaya, The Chiral Condensate in a Finite Volume, JHEP 01 (2009) 052 [arXiv: 0812.2797] [INSPIRE].

[20] C. Aubin and C. Bernard, Pion and kaon masses in staggered chiral perturbation theory, Phys. Rev. D 68 (2003) 034014 [hep-lat/0304014] [INSPIRE].

[21] C. Aubin and C. Bernard, Pseudoscalar decay constants in staggered chiral perturbation theory, Phys. Rev. D 68 (2003) 074011 [hep-lat/0306026] [INSPIRE].

[22] http://www.thep.lu.se/ bijnens/chpt/.

[23] S. Scherer and M.R. Schindler, Quantum chromodynamics and chiral symmetry, Lect. Notes Phys. 830 (2012) 1 [INSPIRE].

[24] S. Scherer and M.R. Schindler, A chiral perturbation theory primer, hep-ph/0505265 [INSPIRE].

[25] J. Bijnens, Chiral perturbation theory beyond one loop, Prog. Part. Nucl. Phys. 58 (2007) 521 [hep-ph/0604043] [INSPIRE].

[26] J. Bijnens, G. Colangelo and G. Ecker, The Mesonic chiral Lagrangian of order $p^{6}$, JHEP 02 (1999) 020 [hep-ph/9902437] [INSPIRE].

[27] J. Bijnens, G. Colangelo, G. Ecker, J. Gasser and M.E. Sainio, Pion pion scattering at low-energy, Nucl. Phys. B 508 (1997) 263 [Erratum ibid. B 517 (1998) 639] [hep-ph/9707291] [INSPIRE].

[28] J. Bijnens, G. Colangelo and G. Ecker, Renormalization of chiral perturbation theory to order p ${ }^{6}$, Annals Phys. 280 (2000) 100 [hep-ph/9907333] [INSPIRE].

[29] M. Golterman, Applications of chiral perturbation theory to lattice QCD, arXiv:0912.4042 [INSPIRE]. 
[30] S.R. Sharpe, Quenched chiral logarithms, Phys. Rev. D 46 (1992) 3146 [hep-lat/9205020] [INSPIRE].

[31] S.R. Sharpe and N. Shoresh, Partially quenched chiral perturbation theory without Phi0, Phys. Rev. D 64 (2001) 114510 [hep-lat/0108003] [INSPIRE].

[32] P.H. Damgaard and K. Splittorff, Partially quenched chiral perturbation theory and the replica method, Phys. Rev. D 62 (2000) 054509 [hep-lat/0003017] [INSPIRE].

[33] J. Bijnens and N. Danielsson, The $\eta$ mass and NNLO three-flavor partially quenched chiral perturbation theory, Phys. Rev. D 74 (2006) 054503 [hep-lat/0606017] [INSPIRE].

[34] D. Becirevic and G. Villadoro, Impact of the finite volume effects on the chiral behavior of $f_{K}$ and $B_{K}$, Phys. Rev. D 69 (2004) 054010 [hep-lat/0311028] [InSPIRE].

[35] G. Amorós, J. Bijnens and P. Talavera, Two point functions at two loops in three flavor chiral perturbation theory, Nucl. Phys. B 568 (2000) 319 [hep-ph/9907264] [INSPIRE].

[36] J. Bijnens, CHIRON: a package for ChPT numerical results at two loops, Eur. Phys. J. C $\mathbf{7 5}$ (2015) 27 [arXiv: 1412.0887] [INSPIRE].

[37] http://www.thep.lu.se/ bijnens/chiron/.

[38] J. Bijnens and G. Ecker, Mesonic low-energy constants, Ann. Rev. Nucl. Part. Sci. 64 (2014) 149 [arXiv: 1405.6488] [INSPIRE].

[39] J.A.M. Vermaseren, New features of FORM, math-ph/0010025 [INSPIRE]. 